\title{
7. GEOCHEMISTRY OF BASALTS FROM HOLE 504B, LEG 83, COSTA RICA RIFT ${ }^{1}$
}

\author{
E. Tual, B. M. Jahn, Université de Rennes, Institut de Geologie \\ H. Bougault, CNEXO-COB \\ and \\ J. L. Joron, Laboratoire "Pierre Sue"2
}

\begin{abstract}
The major element geochemistry of basalts recovered from Leg 83, Hole 504B, shows the typical features of midocean ridge basalts (MORB). The range of variation in their composition, together with the behavior of compatible trace elements $(\mathrm{Co}, \mathrm{Ni}, \mathrm{Cr})$, indicate the well-known relative abundance of minerals that crystallize from these basaltic liquids: plagioclase, olivine, pyroxene, and spinel in decreasing abundance. The hygromagmaphile (or LILE or incompatible) elements are extremely depleted in light rare earths. Nevertheless, some units show flat and enriched REE patterns. These patterns, together with the values of the $\mathrm{La} / \mathrm{Ta}$ ratio, are interpreted in terms of local mantle heterogeneity.
\end{abstract}

\section{INTRODUCTION}

Hole 504B, located about $200 \mathrm{~km}$ south of the Costa Rica Rift in the East Pacific, has been drilled to a total depth of $1350 \mathrm{~m}$ below the seafloor $(1075.5 \mathrm{~m}$ into the basement rocks). The drilling was accomplished by Legs 69 and 70 in 1979 and by Leg 83 in 1981.'Drilling on Leg 83 penetrated the pillow lavas and reached the sheeted dike complex and massive units of Layer 2C (Anderson et al., 1982). At present, 147 lithological units have been identified (see Hole 504B summary chapter, this volume). In this report, we present major and trace element data for 57 basalt samples from different units recovered from the Leg 83 section, 836 to $1350 \mathrm{~m}$ below the seafloor. These samples were selected for their apparent freshness in hand specimen. In addition, 14 samples most representative of the lithologic units from this hole were chosen for precise REE analyses by the isotopic dilution method. The newly acquired geochemical data will be used for petrogenetic discussions.

\section{METHODS}

All major oxides were analyzed by the X-ray fluorescence (XRF) method as described by Bougault (1977). Trace elements such as $\mathrm{Zr}$, $\mathrm{Rb}, \mathrm{Sr}, \mathrm{V}, \mathrm{Y}$, and $\mathrm{Nb}$ were also measured by XRF. Matrix and instrumental effects were corrected following the procedures of Bougault et al. (1977) and, particularly for Nb measurements, Etoubleau et al. (in press). $\mathrm{Sc}, \mathrm{La}, \mathrm{Eu}, \mathrm{Tb}, \mathrm{Hf}, \mathrm{Ta}$, and $\mathrm{Th}$ were determined by neutron activation analysis (NAA) using epithermal neutrons (OSIRIS reactor in Saclay). $\mathrm{Cr}, \mathrm{Co}$, and $\mathrm{Ni}$ were determined by both XRF and NAA methods (Jaffrezic et al., 1977). The analytical method for the 14 samples subjected to precise analysis can be found in Jahn, Auvray, et al. (1980) and Jahn, Griffiths, et al. (1980). The uncertainties were estimated at $3 \%$ for $\mathrm{La}, \mathrm{Lu}$, and $\mathrm{Gd}$, and $2 \%$ for the rest of REE. Similar basalt geochemical data from sections from Legs 69 and 70 are given by Etoubleau et al. (1983), Autio and Rhodes (1983), Marsh et al. (1983), Hubberten et al. (1983), and Natland et al. (1983). The geo-

\footnotetext{
${ }^{1}$ Anderson, R. N., Honnorez, J., Becker, K., et al., Init. Repts. DSDP, 83: Washington (U.S. Govt. Printing Office).

2 Addresses: (Tual; Jahn) CAESS, Université de Rennes, Institut de Geologie, 35042 Rennes, Cedex, France; (Bougault) CNEXO, Centre Océanologique de Bretagne, BP 337, 29273 Brest, Cedex, France; (Joron) Laboratoire d'analyse par activation "Pierre Sue" Cen. Saclay 91191 GIF Sur Yvette, Cedex, France.
}

chemistry of Leg 83 basalts from Hole 504B is also discussed by Emmerman (this volume), Kempton (this volume), and Alt et al. (this volume).

\section{RESULTS}

\section{Major Element Composition}

The major element data are presented in Table 1. It is assumed that most of the basalt samples that have been analyzed can be considered representative of liquid compositions. All samples show relatively homogeneous composition. The composition of the three rocks chosen as reference samples by the shipboard party is given in Table 2.

The range of variation of basalt composition is almost identical to those of the samples of the upper column from Legs 69 and 70 (Etoubleau et al., 1983). No chemical distinction can be made between pillow lavas and dike samples nor between various lithologic units as defined by the shipboard party. A comparison with the data of Leg 54 (EPR $9^{\circ}$ N, Sites 420 to 423,428 , and 429; Joron, Briqueu, et al., 1980) and Leg 65 (EPR $22^{\circ} \mathrm{N}$, Sites 482 and 483, Cambon et al., 1983; Kudo et al., 1982) reveals that for rocks of similar $\mathrm{SiO}_{2}$ content, the basalts of Leg 83 apparently. have lower $\mathrm{TiO}_{2}$ and $\mathrm{P}_{2} \mathrm{O}_{5}$ but are similar to those of the tholeiites from other spreading ridges (MAR, Leg 37, Leg 49, Sites 411 and 412).

Like the majority of ocean floor basalts, the Leg 83 basalts are hypersthene normative and could be called olivine-tholeiites ( $0-16 \%$ olivine in the norm) according to the classification of Yoder and Tilley (1962). Judging from the high $\mathrm{mg}\left(\mathrm{Mg}^{++} /\left(\mathrm{Mg}^{++}+\mathrm{Fe}^{++}\right)\right.$atomic ratio values, 0.62 to 0.70 with a mean of 0.67 , some of the basalts are rather primitive and had experienced only a small degree of fractional crystallization before their eruption or intrusion. Some mg values are close to the most primitive basalts identified to date for MORB (mg = 0.70-0.73; Frey et al., 1974; Langmuir et al., 1977; Donaldson and Brown, 1977; Dungan and Rhodes, 1978; Bougault, 1980; Wilkinson, 1982). In Figure 1, $\mathrm{TiO}_{2}$ 
Table 1. Major element composition (wt.\%) and trace element concentrations (ppm) in Hole 504B basalts.

\begin{tabular}{|c|c|c|c|c|c|c|c|c|c|c|c|c|}
\hline \multirow[b]{3}{*}{$\begin{array}{c}\text { Sample } \\
\text { Interval in } \mathrm{cm} \\
\text { Piece }\end{array}$} & \multicolumn{3}{|c|}{ Leg 69} & \multicolumn{3}{|c|}{ Leg 70} & \multicolumn{6}{|c|}{ Leg 83} \\
\hline & & & & & & & 1 & 2 & 3 & 4 & 5 & 6 \\
\hline & $\begin{array}{c}8-2 \\
125-131\end{array}$ & $\begin{array}{c}18-1 \\
84-98\end{array}$ & $\begin{array}{c}19-1 \\
78-77\end{array}$ & $\begin{array}{c}44-1 \\
25-28\end{array}$ & $\begin{array}{c}56-1 \\
139-142\end{array}$ & $\begin{array}{c}57-1 \\
51-55\end{array}$ & $\begin{array}{c}72-3 \\
41-43 \\
5 \mathrm{a} \\
\end{array}$ & $\begin{array}{c}73-1 \\
43-46 \\
5 \mathrm{a} \\
\end{array}$ & $\begin{array}{c}75-1 \\
119-121 \\
6 \\
\end{array}$ & $\begin{array}{c}77-1 \\
71-74 \\
4\end{array}$ & $\begin{array}{c}77-2 \\
123-126 \\
10\end{array}$ & $\begin{array}{c}78-2 \\
52-54 \\
1 \\
\end{array}$ \\
\hline $\mathrm{SiO}_{2}$ & 49.50 & 48.29 & 48.49 & 49.46 & 49.37 & 49.43 & 48.21 & 48.33 & 48.08 & 48.81 & 48.68 & 47.67 \\
\hline $\mathrm{Ap}_{2} \mathrm{O}_{3}$ & 16.06 & 16.56 & 16.95 & 15.43 & 14.57 & 14.71 & 15.44 & 14.92 & 15.99 & 15.60 & 14.31 & 16.04 \\
\hline $\mathrm{Fe}_{2} \mathrm{O}_{3}$ & 9.26 & 10.02 & 9.28 & 9.80 & 10.53 & 9.99 & 9.58 & 9.63 & 9.72 & 9.92 & 11.11 & 10.05 \\
\hline $\mathrm{MnO}^{2}$ & 0.15 & 0.18 & 0.20 & 0.16 & 0.17 & 0.16 & 0.19 & 0.16 & 0.17 & 0.16 & 0.18 & 0.19 \\
\hline $\mathrm{MgO}$ & 8.05 & 6.80 & 6.39 & 8.27 & 8.54 & 8.43 & 8.54 & 9.15 & 7.86 & 8.35 & 8.44 & 8.47 \\
\hline $\mathrm{CaO}$ & 12.93 & 12.59 & 13.07 & 13.09 & 12.34 & 12.22 & 12.47 & 12.31 & 12.96 & 12.10 & 12.15 & 12.71 \\
\hline $\mathrm{Na}_{2} \mathrm{O}$ & 1.74 & 2.34 & 2.29 & 1.96 & 2.12 & 2.23 & 1.45 & 2.23 & 1.42 & 2.29 & 2.11 & 1.74 \\
\hline $\mathrm{K}_{2} \mathrm{O}$ & 0.02 & 0.39 & 0.15 & 0.07 & 0.05 & 0.11 & $<0.02$ & $<0.02$ & $<0.02$ & $<0.02$ & $<0.02$ & $<0.02$ \\
\hline $\mathrm{TiO}_{2}$ & 0.87 & 1.22 & 1.35 & 0.92 & 1.36 & 1.34 & 0.82 & 0.83 & 0.91 & 0.95 & 1.02 & 0.92 \\
\hline $\mathrm{P}_{2} \mathrm{O}_{5}$ & 0.06 & 0.16 & 0.19 & 0.10 & 0.18 & 0.18 & 0.08 & 0.07 & 0.08 & 0.08 & 0.11 & 0.08 \\
\hline LoI & 1.15 & 1.72 & 1.56 & 0.96 & 0.57 & 1.25 & 1.81 & 1.56 & 1.94 & 1.56 & 1.43 & 2.10 \\
\hline Total & 99.79 & 100.27 & 99.92 & 100.22 & 99.80 & 99.99 & 98.61 & 99.22 & 99.15 & 99.84 & 99.56 & 99.99 \\
\hline $\mathrm{mg} \times 100$ & 63 & 57 & 58 & 62 & 61 & 62 & 68 & 69 & 65 & 66 & 64 & 66 \\
\hline Sc & n.d & 36.5 & 37.2 & 43.3 & 46.5 & 45.8 & 37 & 37.5 & 38.7 & 39.7 & 429 & 39.2 \\
\hline $\mathrm{Ti}$ & 5280 & 7440 & 8220 & 5520 & 8160 & 8040 & 49.80 & 5040 & 5580 & 5760 & 6180 & 5640 \\
\hline V & 284 & 254 & 279 & 314 & 356 & 366 & 241 & 237 & 251 & 228 & 323 & 220 \\
\hline $\mathrm{Cr}$ & 381 & 390 & 400 & 414 & 285 & 357 & 371 & 383 & 370 & 313 & 219 & 318 \\
\hline $\mathrm{Co}^{\mathrm{a}}$ & 39 & 34 & 39 & 43 & 43 & 44 & 44 & 42 & 44 & 40 & 44 & 45 \\
\hline Co & n.d & 35 & 40 & 46 & n.d & n.d & 46 & 46 & 46 & 43 & 44 & 47 \\
\hline $\mathrm{Ni}^{\mathrm{a}}$ & 126 & 110 & 117 & 103 & 86 & 132 & 146 & 152 & 154 & 131 & 86 & 158 \\
\hline $\mathrm{Ni}$ & n.d & 105 & 110 & 102 & n.d & n.d & 170 & 159 & 159 & 122 & 89 & 157 \\
\hline $\mathrm{Rb}$ & $\mathrm{tr}$ & 10.1 & 1.8 & 0.8 & 0.4 & 0.3 & 0.4 & 0.6 & $\mathrm{tr}$ & $\mathrm{tr}$ & tr & $\operatorname{tr}$ \\
\hline $\mathrm{Sr}$ & 71 & 153 & 178 & 62 & 107 & 107 & 63 & 64 & 75 & 75 & 57 & 75 \\
\hline Y & 29 & 28 & 30 & 29 & 37 & 37 & 23 & 24 & 27 & 26 & 29 & 26 \\
\hline $\mathrm{Zr}$ & 47 & 87 & 96 & 49 & 106 & 105 & 46 & 51 & 57 & 60 & 60 & 56 \\
\hline $\mathrm{Nb}$ & 0 & 9.9 & 13.2 & 0.2 & 3 & 4 & 1.1 & 0.2 & 2.1 & 0.1 & 1.7 & 1.1 \\
\hline $\mathrm{Hf}^{\mathrm{a}}$ & $\mathrm{n} \cdot \mathrm{d}$ & 2.20 & 2.47 & 1.41 & 2.54 & n.d & 1.26 & 1.34 & 1.56 & 1.56 & 1.51 & 1.38 \\
\hline $\mathrm{Ta}^{\mathrm{a}}$ & $\mathrm{n} . \mathrm{d}$ & 0.70 & 0.85 & 0.018 & 0.16 & 0.16 & 0.021 & 0.019 & 0.021 & 0.025 & 0.034 & 0.019 \\
\hline $\mathrm{Th}^{\mathrm{a}}$ & n.d & 0.80 & 0.84 & n.d & 0.18 & 0.18 & 0.008 & 0.026 & 0.011 & 0.016 & 0.051 & 0.025 \\
\hline $\mathrm{La}^{\mathrm{a}}$ & n.d & 6.10 & 7.90 & 0.78 & 3.20 & 2.90 & 0.89 & 0.85 & 0.7 & 1 & 1.07 & 0.91 \\
\hline $\mathrm{Ce}^{\mathrm{a}}$ & n.d & n.d & n.d & n.d & n.d & n.d & 4.6 & 3 & 3.9 & 3.5 & 6.6 & n.d \\
\hline $\mathrm{Eu}^{\mathrm{a}}$ & n.d & 1.01 & 1.12 & 0.85 & 1.24 & 1.22 & 0.74 & 0.75 & 0.80 & 0.85 & 0.84 & 0.83 \\
\hline $\mathrm{Tb}^{\mathrm{a}}$ & n.d & 0.57 & 0.62 & 0.54 & 0.72 & 0.72 & 0.48 & 0.49 & 0.51 & 0.17 & 0.59 & 0.52 \\
\hline
\end{tabular}

aNAA

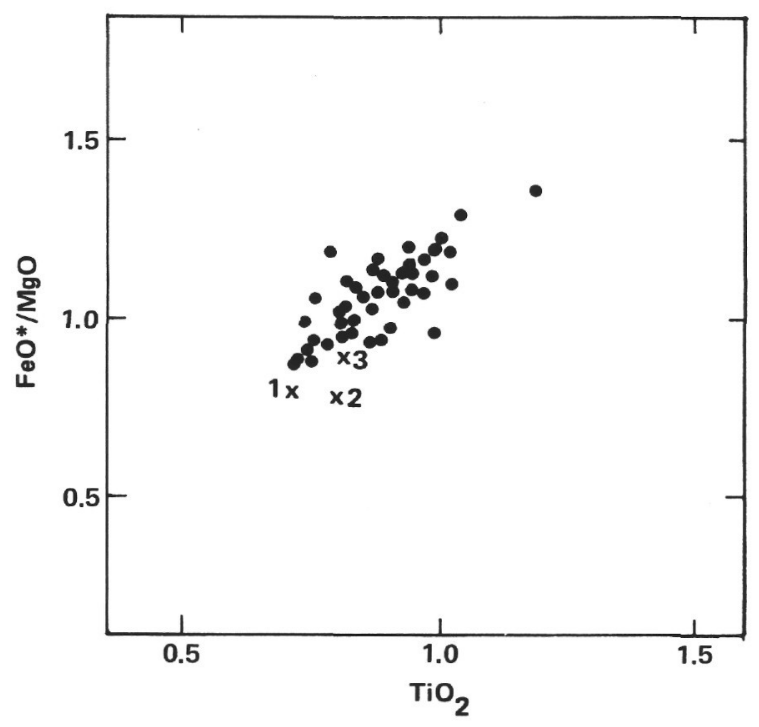

Figure 1. Plot of $\mathrm{TiO}_{2}$ contents (wt.\%) vs. $\mathrm{FeO}^{*} / \mathrm{MgO}$ ratios (all iron as $\mathrm{FeO}$ ) for whole rock analyses and for primary mid-ocean ridge from the literature (1) Frey et al., 1974; (2) Rhodes, Dungan, et al., 1979; (3) Bryan and Moore, 1977. contents are plotted against $\mathrm{FeO} * / \mathrm{MgO}$ ratios (total $\mathrm{Fe}$ as $\mathrm{FeO}$ ) for the present samples. It appears that $\mathrm{TiO}_{2}$ increases with increasing $\mathrm{FeO} * / \mathrm{MgO}$. This positive correlation is consistent with the trend of fractional crystallization and may serve as an indicator of extent of differentiation.

Figures 2 and 3 show that $\mathrm{CaO} / \mathrm{TiO}_{2}$ and $\mathrm{Al}_{2} \mathrm{O}_{3} / \mathrm{TiO}_{2}$ ratios decrease with increasing $\mathrm{TiO}_{2}$. Similarly, the $\mathrm{CaO} /$ $\mathrm{P}_{2} \mathrm{O}_{5}$ and $\mathrm{Al}_{2} \mathrm{O}_{3} / \mathrm{P}_{2} \mathrm{O}_{5}$ ratios decrease with increasing $\mathrm{P}_{2} \mathrm{O}_{5}$ contents. Together with Figure 1, all these correlations could be interpreted as follows:

1. Fractional crystallization. Separation of olivine and pyroxene would lead to a simultaneous increase of the $\mathrm{FeO} / \mathrm{MgO}$ ratio and $\mathrm{TiO}_{2}$ content in the residual liquids, whereas $\mathrm{CaO} / \mathrm{TiO}_{2}$ would decrease. On the other hand, plagioclase separation should lead to a decrease in $\mathrm{Al}_{2} \mathrm{O}_{3} / \mathrm{TiO}_{2}$ as well as $\mathrm{CaO} / \mathrm{TiO}_{2}$ ratios in residual liquids.

2. Various degrees of melting during progressive melting of mantle peridotite. According to Sun et al. (1979), $\mathrm{TiO}_{2}$ would decrease in the melt due to the "dilution" effect. Meanwhile, the phases carrying most of $\mathrm{Al}$ and $\mathrm{Ca}$ such as pyroxene, plagioclase, and spinel become progressively exhausted as the melting advances. Therefore, the $\mathrm{Al}_{2} \mathrm{O}_{3} / \mathrm{TiO}_{2}$ and the $\mathrm{CaO} / \mathrm{TiO}_{2}$ ratios would 
Table 1. (Continued).

\begin{tabular}{|c|c|c|c|c|c|c|c|c|c|c|c|}
\hline \multicolumn{12}{|c|}{ Leg 83} \\
\hline 7 & 8 & 9 & 10 & 11 & 12 & 13 & 14 & 15 & 16 & 17 & 18 \\
\hline $\begin{array}{c}79-1 \\
98-100 \\
17\end{array}$ & $\begin{array}{c}80-3 \\
132-134 \\
8 \mathrm{f} \\
\end{array}$ & $\begin{array}{c}82-3 \\
44-46 \\
5\end{array}$ & $\begin{array}{c}83-2 \\
2-5 \\
1\end{array}$ & $\begin{array}{c}84-1 \\
105-107 \\
6 \\
\end{array}$ & $\begin{array}{c}85-2 \\
113-117 \\
4 a \\
\end{array}$ & $\begin{array}{c}86-1 \\
6-9 \\
1 \\
\end{array}$ & $\begin{array}{c}87-1 \\
93-95 \\
6 \mathrm{~d}\end{array}$ & $\begin{array}{c}88-1 \\
48-50 \\
2 \mathrm{~d} \\
\end{array}$ & $\begin{array}{c}89-1 \\
67-69 \\
7 \\
\end{array}$ & $\begin{array}{c}90-4 \\
69-71 \\
6 c \\
\end{array}$ & $\begin{array}{c}91-1 \\
134-137 \\
18\end{array}$ \\
\hline 47.54 & 47.00 & 49.09 & 48.58 & 48.63 & 48.47 & 49.24 & 49.05 & 47.75 & 47.55 & 49.62 & 48.05 \\
\hline 16.55 & 17.03 & 15.32 & 15.19 & 14.79 & 14.89 & 14.70 & 14.40 & 15.19 & 16.38 & 15.09 & 15.62 \\
\hline 9.89 & 9.58 & 9.50 & 9.23 & 9.35 & 10.10 & 10.70 & 10.88 & 10.35 & 9.60 & 9.67 & 9.89 \\
\hline 0.15 & 0.26 & 0.25 & 0.25 & 0.25 & 0.22 & 0.20 & 0.24 & 0.25 & 0.24 & 0.21 & 0.25 \\
\hline 7.86 & 7.30 & 8.87 & 8.67 & 9.11 & 8.54 & 8.46 & 8.12 & 8.51 & 9.12 & 8.33 & 9.06 \\
\hline 12.96 & 12.98 & 12.50 & 12.66 & 11.84 & 12.86 & 12.66 & 12.91 & 12.31 & 12.35 & 11.11 & 13.23 \\
\hline 1.63 & 2.09 & 1.31 & 1.91 & 1.52 & 1.40 & 1.48 & 1.96 & 2.04 & 1.71 & 1.49 & 1.49 \\
\hline$<0.02$ & $<0.02$ & 0.02 & $<0.02$ & $<0.02$ & $<0.02$ & $<0.02$ & $<0.02$ & $<0.02$ & $<0.02$ & $<0.02$ & $<0.02$ \\
\hline 0.89 & 0.79 & 0.91 & 0.99 & 0.87 & 0.97 & 0.87 & 1 & 1.02 & 0.81 & 0.86 & 0.74 \\
\hline 0.07 & 0.09 & 0.10 & 0.08 & 0.09 & 0.11 & 0.08 & 0.08 & 0.12 & 0.07 & 0.08 & 0.06 \\
\hline 1.94 & 2.47 & 1.56 & 2.05 & 2.15 & 1.96 & 0.70 & 1.40 & 2.70 & 2.20 & 3.10 & 1.90 \\
\hline 99.50 & 99.61 & 99.55 & 99.63 & 98.62 & 99.54 & 99.15 & 100.06 & 100.26 & 100.05 & 99.58 & 100.31 \\
\hline 65 & 64 & 69 & 69 & 70 & 66 & 63 & 64 & 66 & 69 & 67 & 68 \\
\hline 39.8 & n.d & 39.5 & 39.8 & 38 & 42.7 & 41.3 & 42.7 & 40.2 & 39.1 & 37.9 & 38.6 \\
\hline 5460 & 4860 & 5520 & 6060 & 5340 & 5940 & 5280 & 6060 & 6300 & 4980 & 5340 & 4500 \\
\hline 217 & 238 & 279 & 274 & 285 & 289 & 318 & 309 & 271 & 275 & 217 & 268 \\
\hline 340 & 427 & 344 & 314 & 379 & 364 & 325 & 216 & 272 & 346 & 264 & 369 \\
\hline 43 & 47 & 38 & 36 & 41 & 39 & 43 & 43 & 42 & 37 & 35 & 42 \\
\hline 48 & n.d & 42 & 41 & 39 & 44 & 46 & 44 & 43 & 37 & 37 & 45 \\
\hline 155 & 153 & 127 & 95 & 141 & 105 & 97 & 89 & 111 & 122 & 94 & 134 \\
\hline 159 & n.d & 129 & 114 & 129 & 111 & 102 & 86 & 114 & 124 & 102 & 136 \\
\hline tr & $\mathrm{tr}$ & tr & tr & $\mathrm{tr}$ & tr & 2.4 & $\mathrm{tr}$ & $\mathrm{tr}$ & $\mathrm{tr}$ & tr & 0.5 \\
\hline 76 & 60 & 47 & 49 & 45 & 59 & 53 & 47 & 73 & 46 & 59 & 36 \\
\hline 26 & 24 & 26 & 27 & 26 & 29 & 26 & 30 & 27 & 25 & 23 & 23 \\
\hline 67 & 49 & 63 & 54 & 62 & 68 & 58 & 70 & 78 & 54 & 61 & 46 \\
\hline 1.0 & 0.3 & 0.9 & 0 & 0.8 & 0.9 & 1.1 & 1.4 & 3.9 & 0.3 & 1.2 & 0 \\
\hline 1.38 & n.d & 1.34 & 1.43 & 1.38 & 1.54 & 1.26 & 1.48 & 1.76 & 1.14 & 1.31 & 1.07 \\
\hline 0.015 & n.d & 0.017 & 0.022 & 0.021 & 0.033 & 0.02 & 0.029 & 0.23 & 0.016 & 0.033 & 0.015 \\
\hline 0.018 & n.d & 0.027 & 0.015 & 0.025 & 0.026 & 0.016 & 0.035 & 0.22 & 0.018 & 0.037 & 0.011 \\
\hline 1.04 & n.d & 0.9 & 0.88 & 1.14 & 1.29 & 0.98 & 1.03 & 2.75 & 0.7 & 1.04 & 0.52 \\
\hline n.d & n.d & 3.4 & 3.5 & n.d & 5.4 & 4 & 4.2 & 8.3 & 4.4 & 6.1 & n.d \\
\hline 0.92 & n.d & 0.71 & 0.65 & 0.72 & 0.93 & 0.75 & 0.83 & 0.87 & 0.63 & 0.63 & 0.68 \\
\hline 0.50 & n.d & 0.52 & 0.53 & 0.49 & 0.56 & 0.52 & 0.52 & 0.54 & 0.47 & 0.49 & 0.43 \\
\hline
\end{tabular}

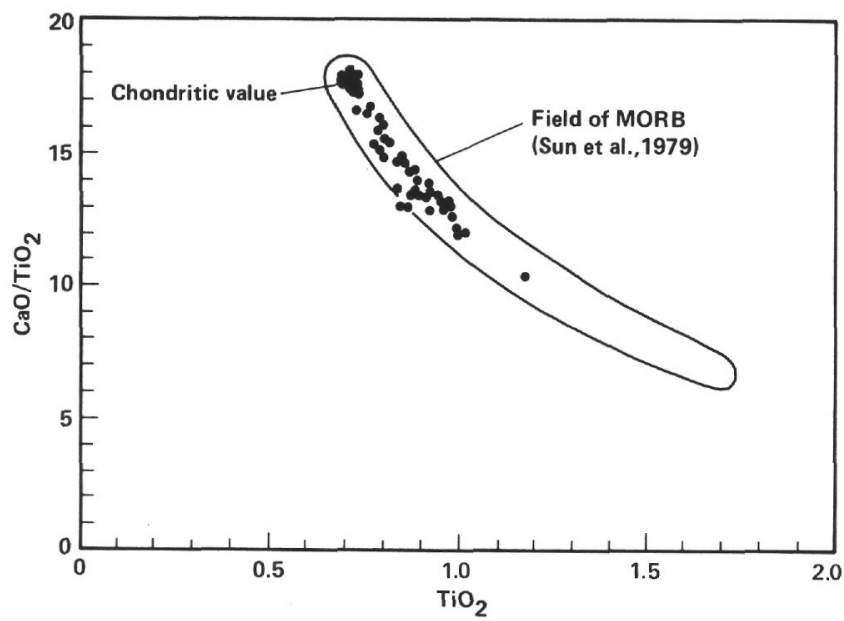

Figure 2. Plot of $\mathrm{CaO} / \mathrm{TiO}_{2}$ ratio vs. $\mathrm{TiO}_{2}$ contents (wt. \%) for whole rock analyses. Chondritic values also reported.

increase in liquids produced from greater degrees of melting.

3. Magma mixing. It is still possible that the correlations were produced by mixing various proportions of at least two end-member magmas, which were perhaps cre-

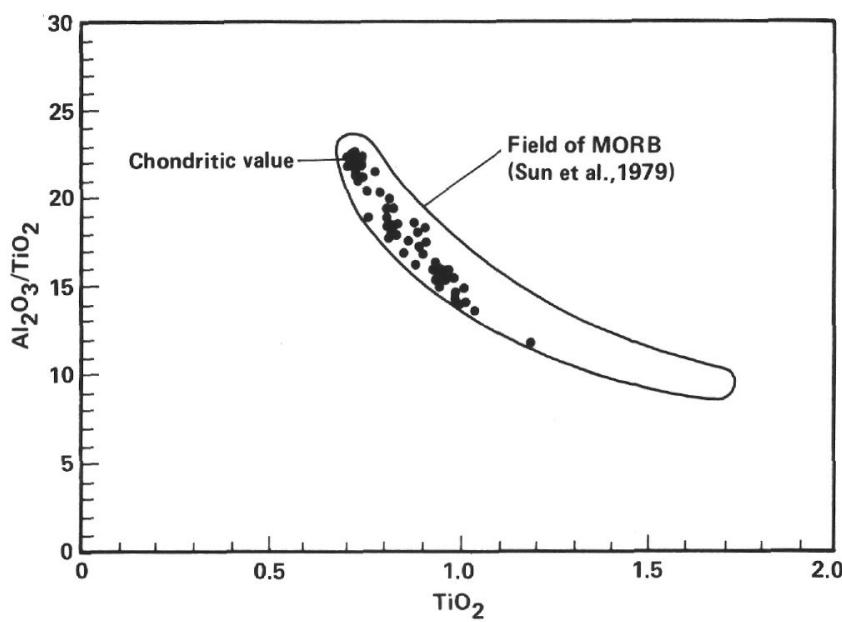

Figure 3. Plot of $\mathrm{Al}_{2} \mathrm{O}_{3} / \mathrm{TiO}_{2}$ ratio vs. $\mathrm{TiO}_{2}$ contents (wt. \%) for whole rock analyses. Chondritic values also reported.

ated earlier by different degrees of melting or by other processes.

In Figures 4 and 5, $\mathrm{CaO}$ and $\mathrm{Al}_{2} \mathrm{O}_{3}$ do not form clear linear correlations with $\mathrm{TiO}_{2}$. In fact, this is expected when a parent magma is subjected to various degrees of 
Table 1. (Continued).

\begin{tabular}{|c|c|c|c|c|c|c|c|c|c|c|c|c|c|}
\hline & 19 & 20 & 21 & 22 & 23 & $\begin{array}{l}\text { Leg } 83 \\
24\end{array}$ & 25 & 26 & 27 & 28 & 29 & 30 & 31 \\
\hline $\begin{array}{c}\text { Sample } \\
\text { Interval in } \mathrm{cm} \\
\text { Piece } \\
\end{array}$ & $\begin{array}{c}92-2 \\
118-121 \\
11 a \\
\end{array}$ & $\begin{array}{c}92-3 \\
64-67 \\
8 \\
\end{array}$ & $\begin{array}{c}93-2 \\
33-35 \\
3\end{array}$ & $\begin{array}{c}94-1 \\
38-41 \\
5\end{array}$ & $\begin{array}{c}94-2 \\
65-67 \\
5\end{array}$ & $\begin{array}{c}94-3 \\
100-103 \\
11\end{array}$ & $\begin{array}{c}97-2 \\
26-29 \\
3 a \\
\end{array}$ & $\begin{array}{c}98-1 \\
75-77 \\
11 \\
\end{array}$ & $\begin{array}{c}99-2 \\
61-63 \\
7\end{array}$ & $\begin{array}{c}100-1 \\
139-144 \\
16\end{array}$ & $\begin{array}{c}101-2 \\
39-43 \\
5\end{array}$ & $\begin{array}{c}102-1 \\
48-51 \\
5 \mathrm{a} \\
\end{array}$ & $\begin{array}{c}104-2 \\
85-87 \\
4 d \\
\end{array}$ \\
\hline $\mathrm{SiO}_{2}$ & 47.37 & 48.82 & 51.21 & 48.92 & 47.72 & 48.97 & 49.62 & 45.64 & 49.39 & 48.25 & 49.71 & 48.51 & 47.28 \\
\hline $\mathrm{Ap}_{2} \mathrm{O}_{3}$ & 15.28 & 14.51 & 14.31 & 15.26 & 15.43 & 15.57 & 14.14 & 16.09 & 14.09 & 16.20 & 14.23 & 15.14 & 16.23 \\
\hline $\mathrm{Fe}_{2} \mathrm{O}_{3}$ & 9.55 & 1.83 & 10.02 & 10.38 & 9.78 & 10.13 & 10.65 & 9.59 & 11.16 & 9.40 & 11.20 & 9.77 & 9.92 \\
\hline $\mathrm{MnO}$ & 0.24 & 0.22 & 0.24 & 0.18 & 0.20 & 0.17 & 0.19 & 0.18 & 0.21 & 0.17 & 0.19 & 0.17 & 0.17 \\
\hline $\mathrm{MgO}$ & 8.45 & 8.10 & 7.83 & 8.20 & 8.67 & 8.46 & 8.24 & 9.25 & 8.23 & 7.73 & 7.85 & 8.81 & 8.16 \\
\hline $\mathrm{CaO}$ & 12.30 & 12.90 & 11.32 & 12.71 & 12.64 & 12.67 & 12.77 & 11.87 & 12.54 & 12.40 & 12.39 & 12.84 & 12.20 \\
\hline $\mathrm{Na}_{2} \mathrm{O}$ & 1.94 & 1.95 & 1.18 & 1.43 & 1.70 & 1.13 & 1.83 & 1.08 & 1.31 & 1.71 & 1.95 & 2.23 & 1.63 \\
\hline $\mathrm{K}_{2} \mathrm{O}$ & $<0.02$ & $<0.02$ & $<0.02$ & $<0.02$ & $<0.02$ & $<0.02$ & $<0.02$ & $<0.02$ & $<0.02$ & $<0.02$ & $<0.02$ & $<0.02$ & $<0.02$ \\
\hline $\mathrm{TiO}_{2}$ & 0.81 & 0.94 & 0.88 & 0.95 & 0.87 & 0.90 & 0.95 & 0.89 & 1 & 0.82 & 1.04 & 0.83 & 0.89 \\
\hline $\mathrm{P}_{2} \mathrm{O}_{5}$ & 0.05 & 0.08 & 0.08 & 0.10 & 0.08 & 0.08 & 0.08 & 0.08 & 0.10 & 0.07 & 0.07 & 0.08 & 0.08 \\
\hline LoI & 2.50 & 1.50 & 1.80 & 1.50 & 2.90 & 1.20 & 1.00 & 3.90 & 1.10 & 1.70 & 1.30 & 1.70 & 2.10 \\
\hline Total & 98.51 & 99.87 & 99.89 & 99.65 & 100.01 & 99.30 & 99.49 & 98.59 & 99.15 & 98.47 & 99.95 & 100.10 & 98.64 \\
\hline $\mathrm{mg} \times 100$ & 67 & 64 & 65 & 65 & 67 & 66 & 64 & 69 & 63 & 66 & 62 & 68 & 66 \\
\hline $\mathrm{Sc}$ & 39.7 & 43.5 & 37.7 & 40.6 & 38.5 & 37.8 & 45.5 & 34.2 & 42.6 & 38.3 & 41.6 & 42.1 & 35.6 \\
\hline $\mathrm{Ti}$ & 4980 & 5700 & 5400 & 5760 & 5400 & 5460 & 5760 & 5580 & 6060 & 498 & 6300 & 5040 & 5460 \\
\hline V & 245 & 281 & 251 & 241 & 240 & 260 & 307 & 215 & 314 & 277 & 318 & 268 & 237 \\
\hline $\mathrm{Cr}$ & 360 & 175 & 294 & 262 & 379 & 337 & 252 & 283 & 222 & 286 & 184 & 390 & 334 \\
\hline $\mathrm{Co}^{\mathrm{a}}$ & 36 & 43 & 42 & 42 & 40 & 41 & 43 & 47 & 43 & 38 & 44 & 41 & 41 \\
\hline Co & 38 & 45 & 41 & 45 & 45 & 45 & 46 & 47 & 46 & 42 & 45 & 46 & 46 \\
\hline $\mathrm{Ni}^{\mathrm{a}}$ & 122 & 101 & 97 & 134 & 132 & 132 & 95 & 222 & 76 & 114 & 78 & 121 & 154 \\
\hline $\mathrm{Ni}$ & 136 & 87 & 96 & 121 & 151 & 131 & 95 & 208 & 92 & 114 & 83 & 123 & 158 \\
\hline $\mathrm{Rb}$ & 0.4 & 0.9 & 0.3 & $\mathrm{tr}$ & $\mathrm{tr}$ & 0.8 & 0.3 & $\mathrm{tr}$ & $\mathrm{tr}$ & $\mathrm{tr}$ & tr & $\operatorname{tr}$ & $\operatorname{tr}$ \\
\hline $\mathrm{Sr}$ & 60 & 47 & 50 & 56 & 56 & 59 & 63 & 57 & 46 & 62 & 52 & 56 & 64 \\
\hline $\mathrm{Y}$ & 25 & 28 & 25 & 26 & 25 & 25 & 28 & 27 & 30 & 24 & 31 & 24 & 25 \\
\hline $\mathrm{Zr}$ & 60 & 64 & 62 & 58 & 56 & 60 & 62 & 73 & 62 & 57 & 58 & 54 & 59 \\
\hline $\mathrm{Nb}$ & 0.6 & 0 & 1 & 0.6 & 0.1 & 0.2 & 1.3 & 1.4 & 1.4 & 0.4 & 0.1 & 0.7 & 1.1 \\
\hline $\mathrm{Hf}^{\mathrm{a}}$ & 1.26 & 1.36 & 1.39 & 1.57 & 1.37 & 1.33 & 1.58 & 1.47 & 1.79 & 1.29 & 1.49 & 1.37 & 1.45 \\
\hline $\mathrm{Ta}^{\mathrm{a}}$ & 0.03 & 0.039 & 0.058 & 0.023 & 0.018 & 0.022 & 0.023 & 0.047 & 0.024 & 0.025 & 0.027 & 0.018 & 0.032 \\
\hline $\mathrm{Th}^{\mathrm{a}}$ & 0.023 & 0.046 & 0.042 & 0.028 & 0.017 & 0.020 & 0.020 & 0.055 & 0.036 & 0.027 & 0.038 & 0.011 & 0.031 \\
\hline $\mathrm{La}^{\mathrm{a}}$ & 1.2 & 1.27 & 1.02 & 0.97 & 0.76 & 0.68 & 0.89 & 1.26 & 1.2 & 1.09 & 0.98 & 0.81 & 1.3 \\
\hline $\mathrm{Ce}^{\mathrm{a}}$ & 4.6 & 4 & 3.5 & 4.1 & 3.4 & 4.1 & n.d & 6.7 & n.d & 2.4 & n.d & 3.2 & 4.7 \\
\hline $\mathrm{Eu}^{\mathrm{a}}$ & 0.73 & 0.81 & 0.69 & 0.91 & 0.69 & 0.78 & 0.94 & 0.83 & 0.86 & 0.71 & 0.91 & 0.68 & 0.79 \\
\hline $\mathrm{Tb}^{\mathrm{a}}$ & 0.48 & 0.52 & 0.66 & 0.53 & 0.49 & 0.52 & 0.52 & 0.52 & 0.58 & 0.49 & 0.67 & 0.49 & 0.51 \\
\hline
\end{tabular}

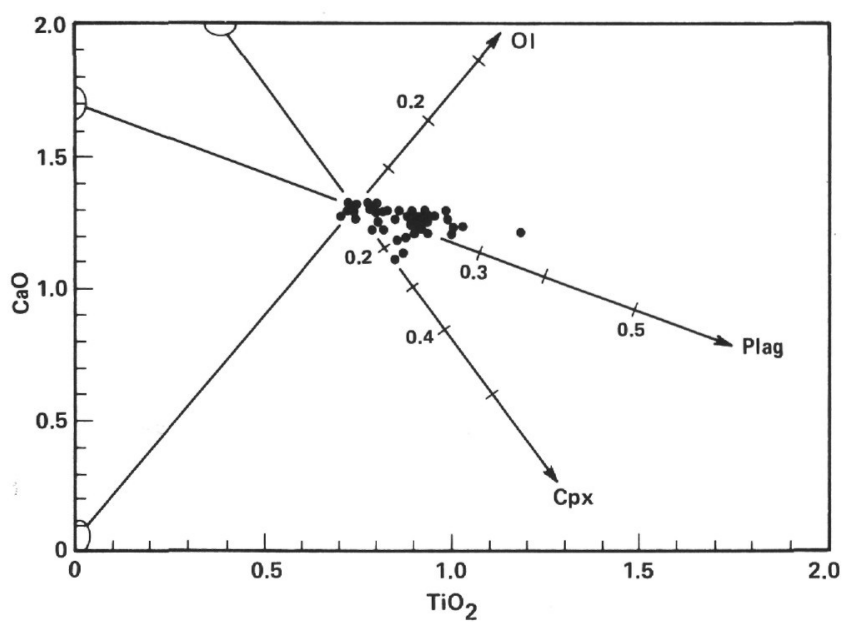

Figure 4. Plot of $\mathrm{CaO}$ contents (wt.\%) vs. $\mathrm{TiO}_{2}$ contents (wt.\%) for whole rock analyses. Common mineral phases composition also reported.

multiphase fractionation. The fractionation trends of different minerals are illustrated to help in understanding the probable proportions of fractionating phases. Graphically depicted, the possible phase assemblage fractionated could be estimated as ol $+\mathrm{plg}+\mathrm{cpx}$ with plg

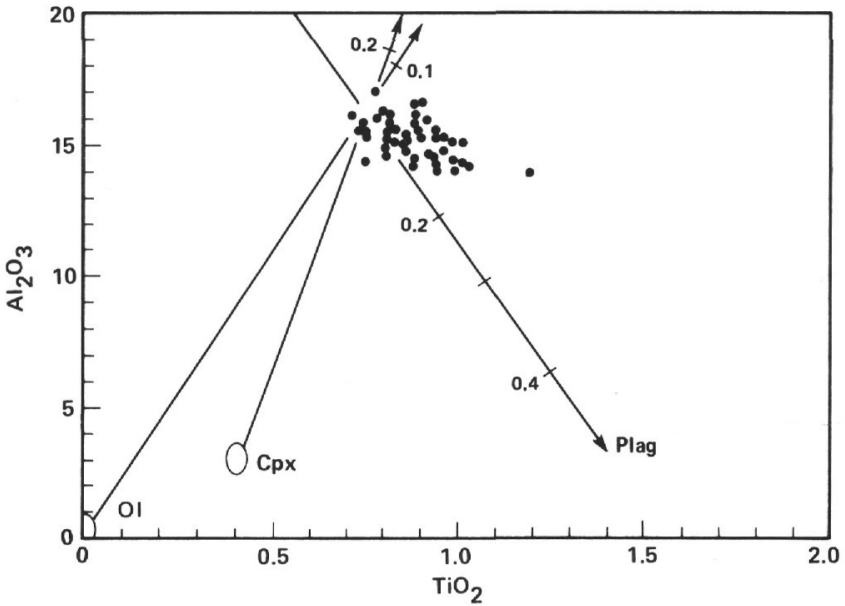

Figure 5. Plot of $\mathrm{Al}_{2} \mathrm{O}_{3}$ contents (wt.\%) vs. $\mathrm{TiO}_{2}$ conients (wt. \%) for whole rock analyses. Common mineral phases composition also reported.

$>$ ol $>\mathrm{cpx}$ in abundance. This is compatible with previous studies of the common crystallization sequence for ocean floor basalts (Bryan et al., 1976); Donaldson and Brown, 1977; Bender et al., 1978; Dungan et al., 1978; Fisk, 1978; Rhodes, Dungan, et al., 1979; Walker 
Table 1. (Continued).

\begin{tabular}{|c|c|c|c|c|c|c|c|c|c|c|c|c|c|}
\hline \multicolumn{14}{|c|}{ Leg 83} \\
\hline 32 & 33 & 34 & 35 & 36 & 37 & 38 & 39 & 40 & 41 & 42 & 43 & 44 & 45 \\
\hline $\begin{array}{c}105-1 \\
61-63 \\
9\end{array}$ & $\begin{array}{c}106-1 \\
35-37 \\
4\end{array}$ & $\begin{array}{c}107-1 \\
116-119 \\
5\end{array}$ & $\begin{array}{c}10-81 \\
57-60 \\
7\end{array}$ & $\begin{array}{c}110-1 \\
34-39 \\
6\end{array}$ & $\begin{array}{c}111-1 \\
86-89 \\
9\end{array}$ & $\begin{array}{c}113-1 \\
68-70 \\
6\end{array}$ & $\begin{array}{c}116-1 \\
24-27 \\
4\end{array}$ & $\begin{array}{c}118-1 \\
33-35 \\
2 \mathrm{c}\end{array}$ & $\begin{array}{c}122-1 \\
116-119 \\
9\end{array}$ & $\begin{array}{c}123-1 \\
39-62 \\
5 a\end{array}$ & $\begin{array}{c}126-1 \\
30-34 \\
4 b\end{array}$ & $\begin{array}{c}127-1 \\
50-53 \\
6\end{array}$ & $\begin{array}{c}128-1 \\
47-49 \\
4 \\
\end{array}$ \\
\hline 48.63 & 48.45 & 49.25 & 49.77 & 48.82 & 44.70 & 48.92 & 49.50 & 50.09 & 48.10 & 48.51 & 48.54 & 48.84 & 48.36 \\
\hline 14.84 & 15.00 & 14.72 & 14.41 & 14.72 & 16.71 & 15.42 & 14.58 & 14.67 & 15.88 & 15.64 & 15.90 & 15.91 & 15.87 \\
\hline 10.10 & 9.67 & 9.57 & 10.02 & 10.37 & 10.10 & 9.64 & 10.36 & 10.44 & 9.34 & 9.95 & 9.11 & 9.13 & 9.38 \\
\hline 0.18 & 0.19 & 0.16 & 0.18 & 0.19 & 0.14 & 0.16 & 0.18 & 0.18 & 0.16 & 0.18 & 0.16 & 0.16 & 0.16 \\
\hline 9.12 & 8.76 & 8.53 & 8.57 & 8.22 & 9.33 & 9.30 & 9.06 & 8.71 & 7.84 & 8.28 & 8.95 & 8.15 & 8.68 \\
\hline 12.74 & 12.90 & 12.72 & 12.60 & 12.40 & 12.16 & 13.13 & 12.72 & 12.64 & 12.72 & 12.91 & 13.01 & 12.99 & 13.15 \\
\hline 2.78 & 1.62 & 1.68 & 1.23 & 2.31 & 1.38 & 1.55 & 1.80 & 1.65 & 1.78 & 1.09 & 1.24 & 1.14 & 1.18 \\
\hline$<0.02$ & $<0.02$ & $<0.02$ & $<0.02$ & $<0.02$ & 0.06 & $<0.02$ & $<0.02$ & $<0.02$ & $<0.02$ & $<0.02$ & $<0.02$ & $<0.02$ & $<0.02$ \\
\hline 0.81 & 0.84 & 0.81 & 0.76 & 0.93 & 0.91 & 0.76 & 0.82 & 0.94 & 0.89 & 0.84 & 0.75 & 0.75 & 0.82 \\
\hline 0.07 & 0.06 & 0.08 & 0.07 & 0.08 & 0.09 & 0.06 & 0.08 & 0.11 & 0.07 & 0.06 & 0.07 & 0.06 & 0.06 \\
\hline 1.10 & 1.80 & 1.30 & 1.00 & 1.40 & 3.80 & 1.00 & 0.70 & 0.85 & 1.90 & 1.20 & 1.10 & 1.20 & 1.30 \\
\hline 100.39 & 99.31 & 98.84 & 98.63 & 99.46 & $99: 44$ & 99.96 & 99.82 & 100.30 & 98.70 & 98.68 & 98.85 & 99.35 & 98.98 \\
\hline 68 & 68 & 68 & 67 & 65 & 68 & 69 & 67 & 66 & 66 & 66 & 70 & 70 & 68 \\
\hline 39 & 43.4 & 39.8 & 42.5 & 41.2 & 36.3 & 38 & 39.4 & 40.4 & 39 & 41 & 36.6 & 36.7 & 37.5 \\
\hline 4920 & 5100 & 4920 & 4620 & 5640 & 5640 & 4620 & 4980 & 5700 & 5460 & 5100 & 4560 & 4980 & 4980 \\
\hline 275 & 279 & 264 & 280 & 287 & 201 & 240 & 298 & 299 & 260 & 248 & 216 & 245 & 245 \\
\hline 360 & 365 & 303 & 285 & 281 & 496 & 346 & 307 & 279 & 358 & 333 & 385 & 335 & 335 \\
\hline 43 & 42 & 42 & 41 & 41 & 53 & 42 & 46 & 42 & 41 & 42 & 41 & 40 & 40 \\
\hline 46 & 44 & 43 & 46 & 44 & 54 & 45 & 46 & 45 & 43 & 45 & 45 & 45 & 45 \\
\hline 126 & 107 & 92 & 103 & 82 & 312 & 136 & 115 & 110 & 125 & 130 & 157 & 144 & 136 \\
\hline 128 & 108 & 99 & 102 & 106 & 295 & 136 & 114 & 104 & 125 & 131 & 159 & 165 & 138 \\
\hline $\mathrm{tr}$ & $\mathrm{tr}$ & $\operatorname{tr}$ & 0.8 & 0.3 & 0.2 & 0.8 & $\mathrm{tr}$ & $\mathrm{tr}$ & $\mathrm{tr}$ & tr & $\mathrm{tr}$ & $\mathrm{tr}$ & tr \\
\hline 55 & 53 & 54 & 58 & 59 & 92 & 51 & 47 & 51 & 68 & 61 & 65 & 61 & 62 \\
\hline 24 & 26 & 23 & 24 & 27 & 25 & 23 & 25 & 28 & 24 & 26 & 22 & 21 & 23 \\
\hline 48 & 49 & 50 & 62 & 57 & 72 & 48 & 50 & 60 & 60 & 57 & 56 & 53 & 58 \\
\hline 1.5 & 1.5 & 1.8 & 0.5 & $\mathrm{tr}$ & 0.7 & 1.1 & 0.5 & 0.8 & 0 & 1.7 & 0 & 0.4 & 0.1 \\
\hline 1.22 & 1.23 & 1.17 & 1.37 & 1.45 & 1.54 & 1.14 & 1.19 & 1.46 & 1.35 & 1.20 & 1.12 & 1.17 & 1.22 \\
\hline 0.019 & 0.011 & 0.02 & 0.023 & 0.02 & 0.048 & 0.018 & 0.014 & 0.011 & 0.021 & 0.026 & 0.018 & 0.021 & 0.026 \\
\hline 0.018 & 0.007 & 0.016 & 0.009 & 0.031 & 0.044 & 0.026 & 0.006 & 0.022 & 0.0008 & 0.027 & 0.035 & 0.033 & 0.019 \\
\hline 0.81 & 0.77 & 0.69 & 0.93 & 1.08 & 1.36 & 0.97 & 0.58 & 0.83 & 0.77 & 0.95 & 0.92 & 0.88 & 0.65 \\
\hline 2.9 & 3.7 & 3.5 & 4 & 3.3 & 6.7 & n.d & 3.5 & 5.4 & 5 & 4 & n.d & 4.1 & $\mathrm{n}$ \\
\hline 0.74 & 0.76 & 0.73 & 0.8 & 0.75 & 0.88 & 0.67 & 0.75 & 0.81 & 0.78 & 0.79 & 0.71 & 0.67 & 0.74 \\
\hline 0.60 & 0.51 & 0.46 & 0.68 & 0.14 & 0.49 & 0.44 & 0.49 & 0.55 & 0.51 & 0.11 & 0.43 & 0.44 & 0.48 \\
\hline
\end{tabular}

et al., 1979). The concentration data of compatible elements (i.e., $\mathrm{Ni}, \mathrm{Cr}, \mathrm{Co}, \mathrm{V}, \mathrm{Sc}$ ) appear to confirm this observation (see below).

\section{Trace Elements}

\section{Compatible Elements}

The concentration ranges of the compatible elements (Co, Ni, Cr) are typical of oceanic tholeiites (Blanchard et al,. 1976; Rhodes, Blanchard, et al., 1979; Johnson, 1979; Wood et al., 1979). In order to estimate the bulk distribution coefficients for these elements, we use the classical relationships deduced from the Rayleigh law (Bougault and Hekinian, 1974):

$$
\log C_{\mathrm{lc}}=\left(1-D_{c}\right) \log C_{l i}+K,
$$

where

$C_{\mathrm{lc}}=$ concentration of a compatible element in the liquid;

$C_{\mathrm{li}}=$ concentration of a hygromagmaphile element in the liquid; and

$D_{\mathrm{c}}=$ bulk partition coefficient of the compatible element.

Therefore, in a log-log plot of the concentration of a compatible element versus that of an incompatible ele- ment, the resulting straight line will have a slope corresponding to $\left(1-D_{c}\right)$, so that $D_{c}$ can be calculated.

Using $\mathrm{Ti}$ as the incompatible element, some compatible elements are plotted in this manner in Figures 6A-C. It has been shown that $\mathrm{Ti}$ in oceanic tholeiites behaves in a similar manner to Sm (Bougault, 1980). Thus, the plots in Figures 6A-C correspond to plots of compatible elements versus the equivalent of $\mathrm{Sm}$. The $K_{D}$ values used here are given in Table 3.

In the samples that we have analyzed, Co varies between 35 and $53 \mathrm{ppm}$ (Fig. 6A); there is a tendency for Co to increase with increasing $\mathrm{Ti}$ content, suggesting that the bulk distribution coefficient for Co is $<1$. Based on our knowledge of mineral melt distribution coefficients for $\mathrm{Co}$, we can calculate the following equation:

$$
\begin{aligned}
D_{\mathrm{Co}} & =\left(X_{\mathrm{sp}} \times 3\right)+\left(X_{\mathrm{plg}} \times 0.1\right)+\left(X_{\mathrm{cpx}} \times 1.0\right) \\
& +\left(X_{\mathrm{ol}} \times 3\right),
\end{aligned}
$$

where $X_{i}=$ weight proportion of phase $i$ that is fractionated from the liquid.

This implies that the percent of separation of olivine or olivine + spinel is not significant, whereas the fractionation of a low $\mathrm{K}_{\mathrm{D}}$ mineral such as plagioclase may be important.

$\mathrm{Ni}$ is expected to partition into olivine and spinel and should be an indicator of olivine \pm spinel fractionation. 
Table 1. (Continued).

\begin{tabular}{|c|c|c|c|c|c|c|c|c|c|c|c|c|}
\hline \multicolumn{13}{|c|}{ Leg 83} \\
\hline & 46 & 47 & 48 & 49 & 50 & 51 & 52 & 53 & 54 & 55 & 56 & 57 \\
\hline $\begin{array}{c}\text { Sample } \\
\text { Interval in } \mathrm{cm} \\
\text { Piece } \\
\end{array}$ & $\begin{array}{c}129-1 \\
35-39 \\
3 \mathrm{~b}\end{array}$ & $\begin{array}{c}129-2 \\
118-121 \\
10\end{array}$ & $\begin{array}{c}130-2 \\
9-12 \\
19\end{array}$ & $\begin{array}{c}131-1 \\
67-70 \\
7\end{array}$ & $\begin{array}{c}132-1 \\
59-61 \\
8 \\
\end{array}$ & $\begin{array}{c}133-1 \\
18-22 \\
3 \\
\end{array}$ & $\begin{array}{c}135-2 \\
69-76 \\
8 \\
\end{array}$ & $\begin{array}{c}134-1 \\
53-57 \\
7\end{array}$ & $\begin{array}{c}136-1 \\
35-40 \\
5\end{array}$ & $\begin{array}{c}137-1 \\
41-47 \\
5\end{array}$ & $\begin{array}{c}138-1 \\
8-12 \\
2 \\
\end{array}$ & $\begin{array}{c}138-1 \\
40-45 \\
6 \\
\end{array}$ \\
\hline $\mathrm{SiO}_{2}$ & 48.23 & 50.03 & 48.46 & 49.11 & 48.98 & 49.54 & 49.20 & 49.00 & 46.30 & 48.96 & 49.13 & 48.85 \\
\hline $\mathrm{Ap}_{2} \mathrm{O}_{3}$ & 16.21 & 14.49 & 15.62 & 15.59 & 15.89 & 14.49 & 14.49 & 15.41 & 14.02 & 16.13 & 15.28 & 15.72 \\
\hline $\mathrm{Fe}_{2} \mathrm{O}_{3}$ & 8.91 & 10.07 & 8.94 & 9.09 & 9.24 & 10.71 & 10.86 & 10.48 & 12.47 & 9.23 & 10.05 & 9.33 \\
\hline $\mathrm{MnO}$ & 0.16 & 0.18 & 0.15 & 0.16 & 0.16 & 0.19 & 0.21 & 0.18 & 0.21 & 0.16 & 0.17 & 0.16 \\
\hline $\mathrm{MgO}$ & 9.19 & 8.44 & 9.11 & 9.33 & 8.79 & 8.49 & 8.19 & 8.09 & 8.29 & 9.02 & 8.57 & 8.63 \\
\hline $\mathrm{CaO}$ & 12.80 & 12.84 & 12.94 & 13.12 & 13.18 & 12.75 & 12.93 & 2.70 & 12.74 & 13.20 & 12.91 & 13.12 \\
\hline $\mathrm{Na}_{2} \mathrm{O}$ & 0.98 & 1.56 & 1.10 & 1.53 & 1.05 & 2.26 & 1.68 & 1.03 & 1.18 & 1.24 & 1.29 & 1.51 \\
\hline $\mathrm{K}_{2} \mathrm{O}^{2}$ & $<0.02$ & $<0.02$ & $<0.02$ & $<0.02$ & $<0.02$ & $<0.02$ & $<0.02$ & $<0.02$ & $<0.02$ & $<0.02$ & $<0.02$ & $<0.02$ \\
\hline $\mathrm{TiO}_{2}$ & 0.72 & 0.89 & 0.73 & 0.76 & 0.81 & 0.95 & 0.99 & 0.97 & 1.19 & 0.79 & 0.87 & 0.81 \\
\hline $\mathrm{P}_{2} \mathrm{O}_{5}$ & 0.06 & 0.06 & 0.05 & 0.06 & 0.07 & 0.12 & 0.14 & 0.14 & 0.14 & 0.15 & 0.11 & 0.15 \\
\hline LoI & 1.60 & 1.00 & 1.30 & 1.10 & 1.20 & 0.79 & 0.81 & 0.77 & 2.13 & 0.90 & 0.60 & 0.82 \\
\hline Total & 98.88 & 99.58 & 98.39 & 99.87 & 99.39 & 100.29 & 99.49 & 98.77 & 98.07 & 99.78 & 98.98 & 99.10 \\
\hline $\mathrm{mg} \times 100$ & 70 & 66 & 70 & 70 & 69 & 65 & 64 & 65 & 61 & 70 & 67 & 68 \\
\hline $\mathrm{Sc}$ & 33.4 & 43.6 & 35.7 & 36.6 & 37.2 & 41.1 & 42.5 & 41.3 & 43.5 & 36.4 & 37.7 & 40.2 \\
\hline $\mathrm{Ti}$ & 4380 & 5400 & 4440 & 4620 & 4920 & 5760 & 6000 & 5880 & 7320 & 4800 & 5340 & 4920 \\
\hline $\mathrm{V}$ & 209 & 284 & 211 & 227 & 245 & 279 & 265 & 259 & 315 & 203 & 210 & 248 \\
\hline $\mathrm{Cr}$ & 415 & 214 & 399 & 382 & 356 & 288 & 284 & 203 & 232 & 347 & 331 & 338 \\
\hline $\mathrm{Co}^{\mathrm{a}}$ & 41 & 42 & 40 & 40 & 40 & 41 & 40 & 39 & 48 & 38 & 39 & 38 \\
\hline Co & 46 & 46 & 45 & 46 & 44 & 45 & 45 & 45 & 49 & 44 & 44 & 46 \\
\hline $\mathrm{Ni}^{\mathrm{a}}$ & 196 & 90 & 166 & 143 & 133 & 88 & 96 & 100 & 95 & 139 & 116 & 112 \\
\hline $\mathrm{Ni}$ & 195 & 97 & 164 & 162 & 139 & 98 & 101 & 94 & 108 & 150 & 135 & 126 \\
\hline $\mathrm{Rb}$ & $\mathrm{tr}$ & $\operatorname{tr}$ & $\mathrm{tr}$ & $\mathrm{tr}$ & $\mathrm{tr}$ & 0.3 & $\mathrm{tr}$ & 0.3 & $\mathrm{tr}$ & $\operatorname{tr}$ & tr & $\operatorname{tr}$ \\
\hline $\mathrm{Sr}$ & 61 & 58 & 61 & 61 & 62 & 45 & 45 & 56 & 45 & 61 & 62 & 60 \\
\hline Y & 20 & 26 & 22 & 22 & 23 & 28 & 29 & 26 & 33 & 21 & 24 & 22 \\
\hline $\mathrm{Zr}$ & 53 & 58 & 49 & 54 & 56 & 55 & 57 & 63 & 72 & 43 & 49 & 52 \\
\hline $\mathrm{Nb}$ & 1.1 & 0.4 & $\mathrm{tr}$ & 0.5 & $\mathrm{tr}$ & 0.7 & 0.3 & 0 & 0.8 & 0 & 1 & 1.6 \\
\hline $\mathrm{Hf}^{\mathrm{a}}$ & 1.05 & 1.43 & 1.15 & 1.14 & 1.23 & 1.37 & 1.56 & 1.56 & 1.91 & 1.16 & 1.3 & 1.47 \\
\hline $\mathrm{Ta}^{\mathrm{a}}$ & 0.018 & 0.026 & 0.022 & 0.025 & 0.024 & 0.021 & 0.029 & 0.024 & 0.035 & 0.028 & 0.025 & 0.029 \\
\hline$T^{\mathrm{a}}$ & 0.026 & 0.026 & 0.016 & 0.026 & 0.03 & 0.022 & 0.033 & 0.018 & 0.018 & 0.034 & 0.014 & 0.029 \\
\hline $\mathrm{La}^{\mathrm{a}}$ & 0.83 & 1.06 & 0.94 & 0.92 & 0.92 & 0.75 & 0.92 & 1.11 & 1.24 & 0.76 & 0.81 & 1.07 \\
\hline $\mathrm{Ce}^{\mathrm{a}}$ & 4.2 & 4.1 & 4 & 3.4 & 3.8 & 3.8 & 3.6 & 4.1 & 5 & n.d & 3.6 & 3.9 \\
\hline $\mathrm{Eu}^{\mathrm{a}}$ & 0.61 & 0.79 & 0.68 & 0.73 & 0.75 & 0.90 & 0.96 & 0.89 & 0.95 & 0.68 & 0.73 & 0.87 \\
\hline $\mathrm{Tb}^{\mathrm{a}}$ & 0.42 & 0.50 & 0.43 & 0.44 & 0.47 & 0.55 & 0.58 & 0.56 & 0.68 & 0.45 & 0.48 & 0.52 \\
\hline $\begin{array}{l}=\left(X_{\mathrm{sp}}\right) \\
+\left(X_{\mathrm{cpx}}\right.\end{array}$ & 9) + & $\mathrm{ol} \times 12$ & $+(X$ & $\times 0$. & & $L$ & $\begin{array}{l}=( \\
+(2\end{array}$ & $\times 1)$ & $\left(X_{\mathrm{ol}}\right.$ & $0.09)$ & $\left(X_{\mathrm{pl}}\right.$ & $\times 0.01)$ \\
\hline
\end{tabular}

values are more likely to lie between 3 and 5 , assuming the parental liquid has $C \mathrm{O}^{\mathrm{Ni}}=250 \mathrm{ppm}$ and $\mathrm{Co}^{\mathrm{Ti}}=$ $4000 \mathrm{ppm}$. The scattering of data points indicate that the investigated basalts possibly do not derive from the same parental liquid, which may in turn imply that the mantle sources are not so homogeneous and/or that the samples investigated represent the results of magma mixing (Rhodes, Dungan, et al., 1979).

In Figure 6C, we have plotted $\log \mathrm{Cr}$ versus (log $\mathrm{Ti}$ ). Chromium is expected to partition into clinopyroxene and spinel.

$$
\begin{aligned}
D_{\mathrm{Cr}}\left(X_{\mathrm{sp}} \times 60\right) & +\left(X_{\mathrm{ol}} \times 1\right)+\left(X_{\mathrm{plg}} \times 0.02\right) \\
& +\left(X_{\mathrm{cpx}} \times 10\right) .
\end{aligned}
$$

As for $\mathrm{Ni}$, the data are scattered, giving a range of $\mathrm{D}$ values from 2 to 4 if $\mathrm{Co}^{\mathrm{Cr}}=500 \mathrm{ppm}$ is assumed. Certainly the spinel fractionation cannot be very important. Because the partition coefficient of $\mathrm{Cr}$ in clinopyroxene is very high ( $>10$; Bougault, 1980), the fractionation of clinopyroxene cannot be very important, even if it is greater than the fractionation of spinel.

$\mathrm{V}$ and $\mathrm{Sc}$ are expected to partition into clinopyroxene and should be indicators of this mineral fractionation.

However, there is a tendency for $\mathrm{V}$ to increase (Fig. 6D) with increasing $\mathrm{TiO}_{2}$ content, thus suggesting $D_{\mathrm{V}}<1$ and that the effects of fractionation of plagioclase and olivine overshadow those of clinopyroxene. A similar behavior may have also governed Sc. The abundancy of $\mathrm{Sc}$ is nearly constant (Fig. 6E); there is also a tendency to increase slightly with degree of fractionation, which suggests that the bulk partition coefficient is close to unity. This implies that pyroxene fractionation is not an important mechanism.

These observations can be summarized as follows:

1. From Co-Ti data: plagioclase fractionation is very important and olivine \pm spinel is not very significant.

2. From Ni-Ti data: olivine \pm spinel fractionation must exist but plagioclase fractionation can be important.

3. From Cr-Ti data: spinel fractionation is relatively unimportant; clinopyroxene fractionation is present.

4. From V-Ti data: plagioclase and olivine fractionation overshadow that of clinopyroxene.

5. From Sc-Ti data: clinopyroxene fractionation is not an important mechanism. 
Table 2. Major and trace element concentrations for 3 rocks from Hole 504B chosen as reference samples.

\begin{tabular}{|c|c|c|c|}
\hline & $\begin{array}{c}77-1 \\
44-53\end{array}$ & $\begin{array}{c}97-2 \\
74-89\end{array}$ & $\begin{array}{c}130-2 \\
44-58\end{array}$ \\
\hline $\mathrm{SiO}_{2}$ & 47.96 & 50.04 & 48.70 \\
\hline $\mathrm{Ap}_{2} \mathrm{O}_{3}$ & 15.90 & 14.40 & 16.15 \\
\hline $\mathrm{Fe}_{2} \mathrm{O}_{3}$ & 9.99 & 10.82 & 9.20 \\
\hline $\mathrm{MnO}$ & 0.18 & 0.18 & 0.15 \\
\hline $\mathrm{MgO}$ & 8.58 & 8.69 & 9.38 \\
\hline $\mathrm{CaO}$ & 12.59 & 12.93 & 13.32 \\
\hline $\mathrm{Na}_{2} \mathrm{O}$ & 1.74 & 1.41 & 1.36 \\
\hline $\mathrm{K}_{2} \mathrm{O}$ & 0.02 & $<0.02$ & $<0.02$ \\
\hline $\mathrm{TiO}_{2}$ & 0.93 & 0.98 & 0.76 \\
\hline $\mathrm{P}_{2} \mathrm{O}_{5}$ & 0.10 & 0.10 & 0.09 \\
\hline LoI & 1.32 & 0.48 & 0.95 \\
\hline Total & 99.31 & 100.03 & 100.06 \\
\hline $\mathrm{mg} \times 100$ & 67 & 66 & 70 \\
\hline Sc & 39.5 & 45 & 36.5 \\
\hline $\mathrm{Ti}$ & 5580 & 5880 & 4560 \\
\hline V & 235 & 300 & 221 \\
\hline $\mathrm{Cr}$ & 342 & 280 & 441 \\
\hline $\mathrm{Co}^{\mathrm{a}}$ & 40 & 40 & 40 \\
\hline Co & 44 & 46 & 44 \\
\hline $\mathrm{Ni}^{\mathrm{a}}$ & 138 & 84 & 154 \\
\hline $\mathrm{Ni}$ & 128 & 97 & 158 \\
\hline $\mathrm{Rb}$ & $\operatorname{tr}$ & 0.1 & $\mathrm{tr}$ \\
\hline $\mathrm{Sr}$ & 78 & 65 & 63 \\
\hline $\mathrm{Y}$ & 26.5 & 26.9 & 21.4 \\
\hline $\mathrm{Zr}$ & 55 & 64 & 48 \\
\hline $\mathrm{Nb}$ & 1.1 & 1.1 & 1.5 \\
\hline $\mathrm{Hf}^{\mathrm{a}}$ & 1.41 & 1.49 & 1.21 \\
\hline $\mathrm{Ta}^{\mathrm{a}}$ & 0.052 & 0.025 & 0.043 \\
\hline $\mathrm{Th}^{\mathrm{a}}$ & 0.018 & 0.023 & 0.012 \\
\hline $\mathrm{La}^{\mathrm{a}}$ & 0.85 & 1.05 & 0.95 \\
\hline $\mathrm{Ce}^{\mathrm{a}}$ & 5.5 & 5.8 & 2.8 \\
\hline $\mathrm{Eu}^{\mathrm{a}}$ & 0.87 & 0.91 & 0.69 \\
\hline $\mathrm{Tb}^{\mathrm{a}}$ & 0.54 & 0.59 & 0.45 \\
\hline
\end{tabular}

It is concluded that the most important fractionated phase is plagioclase which, in turn, is followed by olivine, clinopyroxene, and spinel.

\section{Incompatible Elements or Hygromagmaphile Elements}

According to the Rayleigh law, a plot of two hygromagmaphile elements will result in a straight line passing through the origin with a slope governed by the initial ratio of the element concentrations in the liquid. This line should be unique (single initial ratio) provided that all samples are cogenetic.

It has been shown that transition metal elements such as $\mathrm{Y}, \mathrm{Zr}$, and $\mathrm{Nb}$ (second series) and $\mathrm{La}, \mathrm{Tb}, \mathrm{Hf}$, and $\mathrm{Tc}$ (third series) have similar $\mathrm{K}_{\mathrm{D}}$ values (Bougault, Treuil, et al., 1979). Sun et al. (1979) and Bougault (1980) have established the order of compatibility of trace elements in basaltic magma as follows (in the order of decreasing $\mathrm{K}_{\mathrm{D}}$ values):

$\mathrm{Lu}-\mathrm{Yb}-\mathrm{Er}-\mathrm{Y}-\mathrm{Dy}-\mathrm{Tb}-\mathrm{Gd}-\mathrm{Eu}(\mathrm{Ti})-$ $\mathrm{Sm}(\mathrm{Zr}-\mathrm{Hf})-\mathrm{Nd}-\mathrm{Ce}-\mathrm{La}-(\mathrm{Nb}-\mathrm{Ta})-\mathrm{Th}$.
The values of the $\mathrm{Y} / \mathrm{Tb}, \mathrm{Zr} / \mathrm{Hf}$, and $\mathrm{Nb} / \mathrm{Ta}$ ratios are given in Table 4 for the North Atlantic basaltic samples and in chondrites.

In Figure 7, Hole 504B samples cluster in the upper part of the range defined by the Atlantic Ocean samples (as do Legs 54 and 65 samples) given a $\mathrm{Y} / \mathrm{Tb}$ ratio close to $50 \pm 3$, which is not statistically different from the presently accepted mean value of $\mathbf{4 6}$ for chondrites (Bougault, 1980). This small discrepancy observed for the $\mathrm{Y} / \mathrm{Tb}$ ratio is probably a product of the analysis. In Figure 8, samples appear in the range of the Atlantic Ocean samples (Bougault, Cambon, et al., 1979) showing a similar dispersion of the $\mathrm{Zr} / \mathrm{Hf}$ ratios (38 and 40 ). In these two figures, the concentrations of these elements for the majority of Hole 504B samples are very low. A plot of $\mathrm{Nb} / \mathrm{Ta}$ is shown in Figure 9. Most concentrations found for these elements are close to the detection limit of XRF techniques. The determination of low $\mathrm{Nb}$ concentration by XRF techniques is accurate to about $30 \%$. For this reason it is not possible to calculate a $\mathrm{Nb} / \mathrm{Ta}$ ratio from a single analysis if the sample has an $\mathrm{Nb}$ concentration of between -1 and $3 \mathrm{ppm}$. There is no ambiguity for the larger concentrations encountered in four samples from Leg $69(18-1 ; 18-2 ; 19-1 ; 19-2)$, three samples from Leg $70(54-1 ; 56-1 ; 57-1)$ and one sample from Leg 83 (88-1) whose average fits the value $16 \pm 1$ found in oceanic basalts and in chondrites, indicating that this ratio is also independent of any mantle heterogeneity. (Note: As Hole 504B is the only hole under consideration the hole number has been eliminated from sample designations. They should be read as core, section, range in $\mathrm{cm}$ ). The important aspect is that the ratios $\mathrm{Y} / \mathrm{Tb}, \mathrm{Zr} / \mathrm{Hf}$, and $\mathrm{Nb} / \mathrm{Ta}$ are nearly constant for a large variation of concentration, suggesting that they do not fractionate during magma evolution. It can be deduced from these results that the mantle is homogeneous with respect to these ratios and close to chondritic composition.

A plot of La versus Ta for North Atlantic samples defines two different correlation lines (Fig. 10). The line with a slope of 19 includes samples recovered from $22^{\circ}$ and $25^{\circ} \mathrm{N}$; the other line corresponds to hot spot samples (i.e., Azores Triple Junction) and alkali basalts (Bougault et al., 1979; Bougault, 1980). The two different slopes suggest that two initial concentration ratios exist. This further implies the derivation of parental liquids from heterogeneous mantle sources in terms of $\mathrm{La} / \mathrm{Ta}$ ratios. The higher La/Ta ratio of approximately 19 corresponds to a characteristic "depleted" oceanic mantle, and the lower ratio of 9 corresponds to an "enriched" mantle. Most of the samples of Hole 504B have low La and Ta concentrations; hence, it is difficult to establish precise $\mathrm{La} / \mathrm{Ta}$ ratios. However, most data in Figure 10 seem to be close to the line corresponding to $\mathrm{La} / \mathrm{Ta}=19$. Nevertheless, four samples from Leg 69 (504B-18-1; 504B$18-2$, ; 504B-19-1, 504B-19-2), three samples from Leg 70 (504B-54-1; 504B-56-1; 504B-57-1), and one sample from Leg 83 (504B-88-1) have higher concentrations, and their $\mathrm{La} / \mathrm{Ta}$ ratios can be determined with greater 


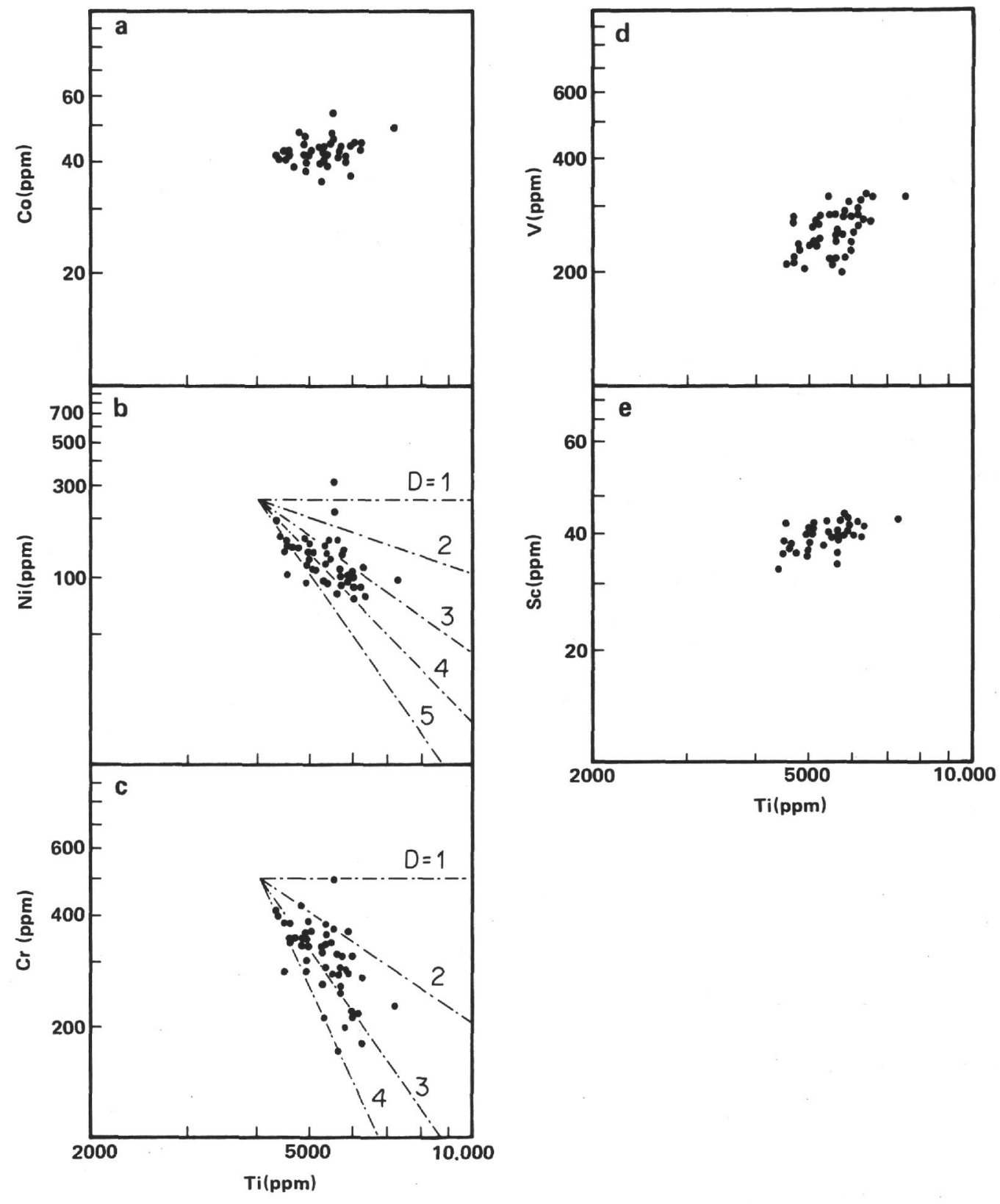

Figures 6A-E. Co, Ni, Cr, V, and Sc (ppm) vs. Ti (ppm) for whole rock analyses. We have also reported lines corresponding to different bulk distribution coefficients from a hypothetical parental liquid.

precision. Among the data points plotted in Figure 10, only three samples yield a value of about 9 , whereas all other samples are found close to the line of 19. This is a very important result because it is the first time that two distinct $\mathrm{La} / \mathrm{Ta}$ ratios have been found in a single hole.

Similarly, a diagram using an incompatible element ratio plot may be used to distinguish different source regions (e.g., Joron, Treuil, et al., 1980).

Fractional crystallization will not substantially alter the elemental ratios, therefore the linear arrays as shown in Figure 11 should represent the chemical characteristics of their source regions.

In Figure 11, available data from Hole 504B and other regions of the East Pacific Rise and North Atlantic
Ocean are plotted. The majority of the basalts from Hole 504B are very LREE depleted (Th/Hf very low) compared to other sites in the Pacific or Atlantic oceans. However, there are some exceptions: Samples 18-1, 18-2, 19-1, and 19-2 (Leg 69) are similar to Leg 49, Site 412 samples. Samples 54-1, 56-1, and 57-1 (Leg 70) are close to Legs 45,46 , and 65 samples. Sample $88-1$ is similar to Leg 49 , Site 409 basalts. Consequently, a large variation of $\mathrm{Th} / \mathrm{Hf}$ ratio found in a single hole enhances the probability of local mantle heterogeneity.

\section{REE Concentrations}

Fourteen samples were selected for precise REE analysis by the isotopic dilution method. The results are shown 
Table 3. Distribution coefficients of $\mathrm{Co}, \mathrm{Ni}, \mathrm{Cr}, \mathrm{V}$, and $\mathrm{Sc}$ in basaltic magmas.

\begin{tabular}{|c|c|c|c|c|c|c|c|c|c|c|}
\hline & Co & Ref & $\mathrm{Ni}$ & Ref & $\mathrm{Cr}$ & Ref & $\mathrm{V}$ & Ref & $\mathrm{Sc}$ & Ref \\
\hline \multirow[t]{3}{*}{ Olivine } & $2-4.5$ & $\begin{array}{l}\text { Leeman, } 1974 \\
\text { Duke, } 1976\end{array}$ & 12 & $\begin{array}{l}\text { Hart and } \\
\text { Davis, 1978; } \\
\text { Bougault and } \\
\text { Hekinian, } 1974\end{array}$ & $0.85 \pm 0.15$ & $\begin{array}{l}\text { Akella et } \\
\text { al., } 1976\end{array}$ & 0.09 & $\begin{array}{l}\text { Bougault and } \\
\text { Hekinian, } 1974\end{array}$ & 0.15 & $\begin{array}{l}\text { Sun and } \\
\text { Nesbitt, } \\
1978\end{array}$ \\
\hline & $3-5$ & $\begin{array}{l}\text { Muir et al., } 1964 \\
\text { Dale and } \\
\text { Henderson, 1972; } \\
\text { Frey et } \\
\text { al., } 1974\end{array}$ & $10-20$ & $\begin{array}{l}\text { Dale and } \\
\text { Henderson, } 1972 \\
\text { Thompson } \\
\text { et al., 1972; } \\
\text { Frey et al., } \\
1974\end{array}$ & 1 & $\begin{array}{l}\text { Bougault } \\
\text { and } \\
\text { Hekinian, } \\
\text { 1974; }\end{array}$ & & $\begin{array}{l}\text { Taylor et } \\
\text { al., 1969; } \\
\text { Frey et } \\
\text { al., } 1978 \\
\quad .\end{array}$ & 0.25 & $\begin{array}{l}\text { Taylor et } \\
\text { al., 1969; } \\
\text { Frey et } \\
\text { al., } 1978\end{array}$ \\
\hline & 3 & $\begin{array}{l}\text { Bougault and } \\
\text { Hekinian, } 1974\end{array}$ & & & & & & - & & \\
\hline \multirow[t]{2}{*}{ Clinopyroxene } & $0.5-1.5$ & $\begin{array}{l}\text { Lindstrom and } \\
\text { Weill, 1978; }\end{array}$ & $1-4$ & $\begin{array}{l}\text { Gast, 1968; } \\
\text { Wager and } \\
\text { Mitchell, } 1951\end{array}$ & $5-10$ & $\begin{array}{l}\text { Gast, 1968; } \\
\text { Schrebier and } \\
\text { Haskin, } 1976\end{array}$ & $<2$ & $\begin{array}{l}\text { Duke, } 1976 \\
\text { Muir et } \\
\text { al., } 1964 \\
\text { Frey et } \\
\text { al., } 1974\end{array}$ & 3 & $\begin{array}{l}\text { Muir et } \\
\text { al., 1964; } \\
\text { Onuma et } \\
\text { al., } 1968\end{array}$ \\
\hline & $0.6-1.3$ & $\begin{array}{l}\text { Bougault and } \\
\text { Hekinian,1974; } \\
\text { Taylor et al., 1969; } \\
\text { Frey et al., } 1978\end{array}$ & $2-4$ & $\begin{array}{l}\text { Bougault and } \\
\text { Hekinian; } 1974\end{array}$ & 13 & $\begin{array}{l}\text { Bougault and } \\
\text { Hekinian, } 1974\end{array}$ & 1.5 & $\begin{array}{l}\text { Taylor et } \\
\text { al., 1969; } \\
\text { Frey et } \\
\text { al., } 1978\end{array}$ & & $\begin{array}{l}\text { Sun and } \\
\text { Nesbitt, } \\
\text { 1978; Frey } \\
\text { et al., } 1974\end{array}$ \\
\hline Plagioclase & 0.1 & $\begin{array}{l}\text { Bougault and } \\
\text { Hekinian, } 1974\end{array}$ & 0.06 & $\begin{array}{l}\text { Bougault and } \\
\text { Hekinian, } 1974\end{array}$ & 0.02 & $\begin{array}{l}\text { Bougault and } \\
\text { Hekinian, } 1974\end{array}$ & & $\begin{array}{l}\text { Bougault and } \\
\text { Hekinian, } 1974\end{array}$ & & $\begin{array}{l}\text { Taylor, et } \\
\text { al.. } 1969\end{array}$ \\
\hline \multirow[t]{2}{*}{ Spinel } & $2-3$ & Bird, 1971 & $10-50$ & $\begin{array}{l}\text { Mysen and } \\
\text { Kushiro, } 1976\end{array}$ & $50-100$ & $\begin{array}{l}\text { Akella et } \\
\text { al., } 1976\end{array}$ & 0.1 & $\begin{array}{l}\text { Bougault and } \\
\text { Hekinian, } 1974\end{array}$ & 0.02 & $\begin{array}{l}\text { Sun and } \\
\text { Nesbitt, } \\
1978\end{array}$ \\
\hline & $4-5$ & $\begin{array}{l}\text { Gast, 1968; Evans, 1969; } \\
\text { Haku and Wright, 1967; } \\
\text { Bougault and } \\
\text { Hekinian, } 1974\end{array}$ & 11 & $\begin{array}{l}\text { Gast, 1968; } \\
\text { Bougault and } \\
\text { Hekinian, 1974; } \\
\text { Evans, 1969; } \\
\text { Haku and } \\
\text { Wright, } 1967\end{array}$ & & & 1.3 & $\begin{array}{l}\text { Gast, 1968; } \\
\text { Evans, 1969; } \\
\text { Haku and } \\
\text { Wright, 1967; } \\
\text { Taylor et } \\
\text { al., } 1969\end{array}$ & & \\
\hline
\end{tabular}

Table 4. $\mathrm{Y} / \mathrm{Tb}, \mathrm{Zr} / \mathrm{Hf}$, and $\mathrm{Nb} / \mathrm{Ta}$ ratio values in North Atlantic basalt samples and in chondrites.

\begin{tabular}{lccc}
\hline & $\mathrm{Y} / \mathrm{Tb}$ & $\mathrm{Zr} / \mathrm{Hf}$ & $\mathrm{Nb} / \mathrm{Ta}$ \\
\hline Basalts & $40-50$ & $39 \pm 5$ & $17 \pm 2$ \\
Chondrites & 46 & $30-40$ & $15-17$ \\
\hline
\end{tabular}

Note: From Bougault, Joron, et al., 1979.

in Table 5 and the chondrite-normalized patterns are displayed in Figure 12. The major observations are as follows:

1. Except for five samples, all REE patterns show distinct REE depletion with $(\mathrm{La} / \mathrm{Sm})_{\mathrm{N}}=0.30-0.48$. This degree of depletion is more pronounced than typical "normal" MORB with $(\mathrm{La} / \mathrm{Sm})_{\mathrm{N}}=0.4-0.7$ (Schilling et al., 1975; Sun et al., 1979; Jahn, Griffiths, et al., 1980) but is comparable to samples from Leg 3 (Sites 14 and 18; Frey et al., 1974). The strong LREE depletion is associated with low $\mathrm{Nb}, \mathrm{Ta}$, and $\mathrm{Th}$ concentrations.

2. $(\mathrm{La})_{\mathrm{N}}$ values are about $3.5 \mathrm{x}$ whereas $(\mathrm{HREE})_{\mathrm{N}}$ are 10-14x chondrite abundances.

3. They show either negative or no Eu anomalies suggesting that plagioclase has played a role in some samples during fractional crystallization. However, samples from Unit 5 of Leg 69 (28-1; 19-1), Unit 36 of Leg 70 (50-1; 51-1), and Unit 69 of Leg 83 (88-1) show different REE abundance patterns. Two samples from Unit 5 show

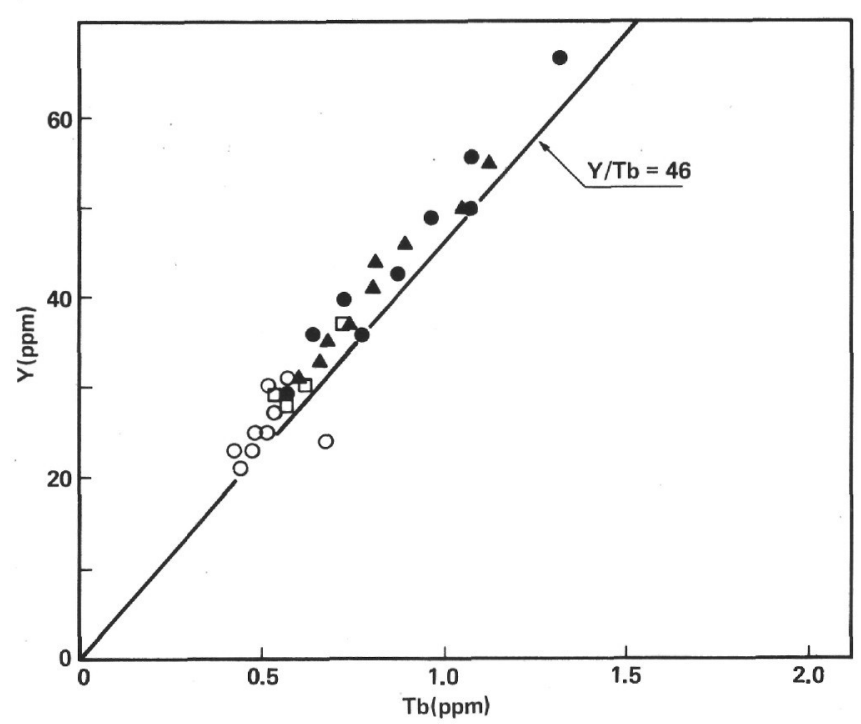

Figure 7. Y (ppm) vs. Tb (ppm). We have reported some samples from the Pacific Ocean: Legs 69 and 70, Hole 504B ( $\square$ ); Leg 83, Hole 504B (O); Leg 65, Holes 482 and $483(\Delta)$; Leg 54, Holes 420 to 423,428 , and $429(\bigcirc)$. Y/Tb ratio reported corresponds to chondritic value.

LREE-enriched patterns with $(\mathrm{La} / \mathrm{Sm})_{\mathrm{N}}=1.60$. This characteristic is similar to that of T-type MORB (Schilling, 1975; White and Schilling, 1978). On the other hand, the samples from Units 36 and 69 have moder- 


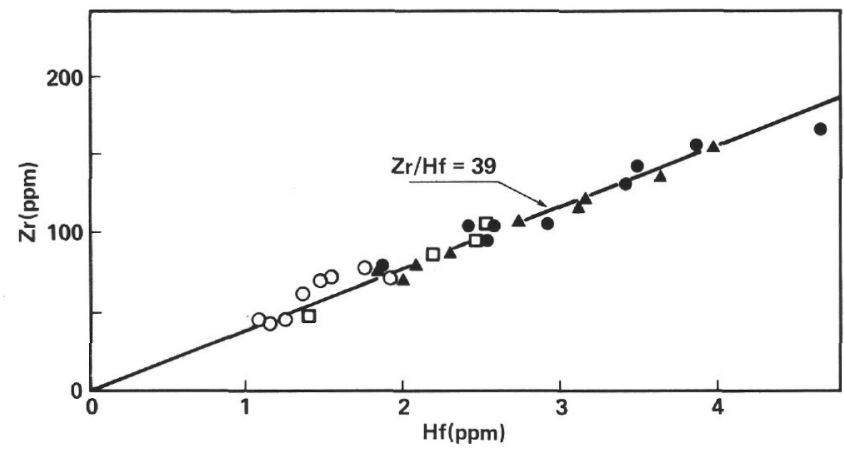

Figure 8. $\mathrm{Zr}$ (ppm) vs. $\mathrm{Hf}$ (ppm). $\mathrm{Zr} / \mathrm{Hf}$ ratio reported corresponds to chondritic value. Same symbols as Figure 7.

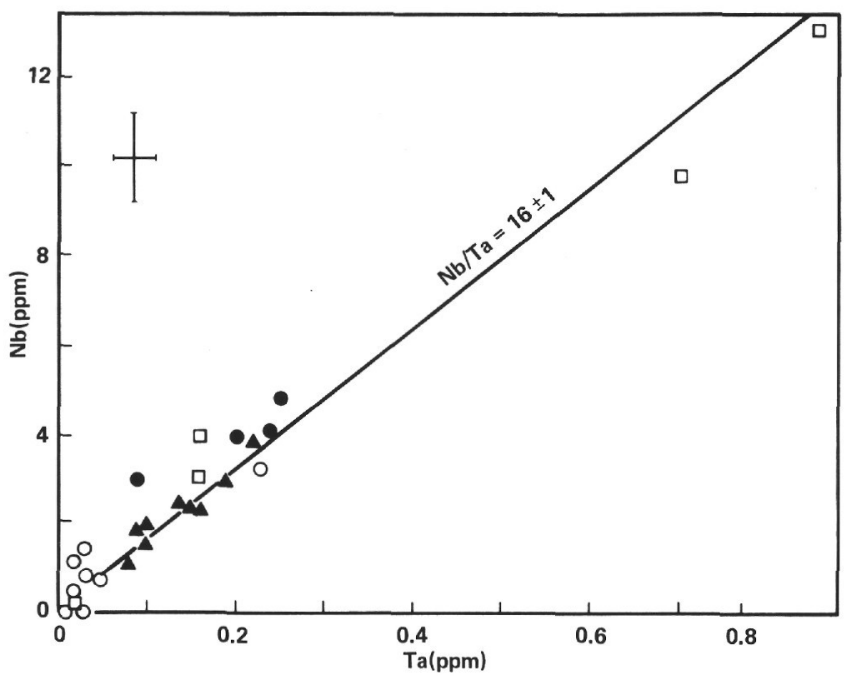

Figure 9. $\mathrm{Nb}(\mathrm{ppm})$ vs. $\mathrm{Ta}(\mathrm{ppm}) . \mathrm{Nb} / \mathrm{Ta}$ ratio reported corresponds to values for chondritic and North Atlantic oceanic basalts. Same symbols as Figure 7.

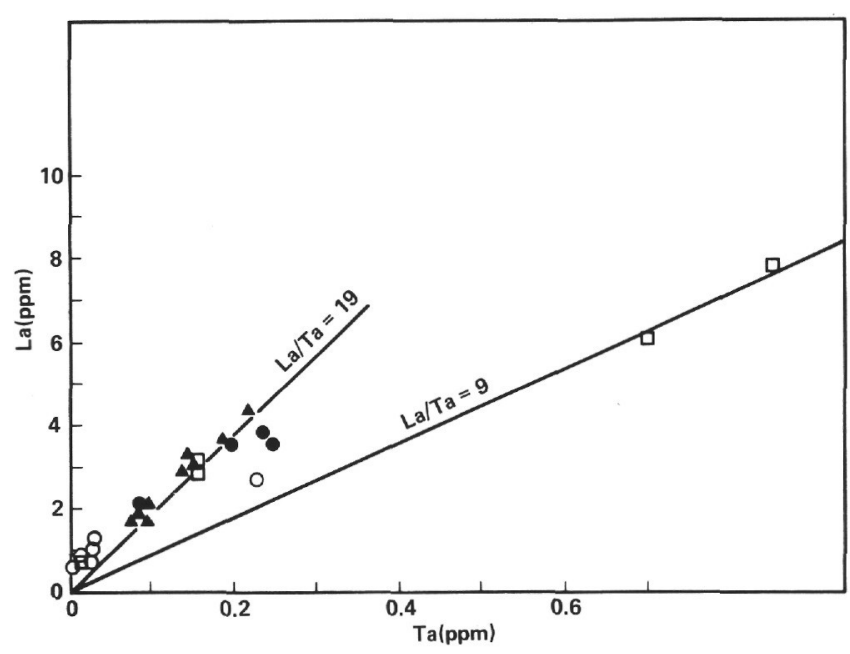

Figure 10. $\mathrm{La}(\mathrm{ppm})$ vs. Ta (ppm). La/Ta ratios correspond to 2 different values found for North Atlantic oceanic basalts. Same symbols as Figure 7.

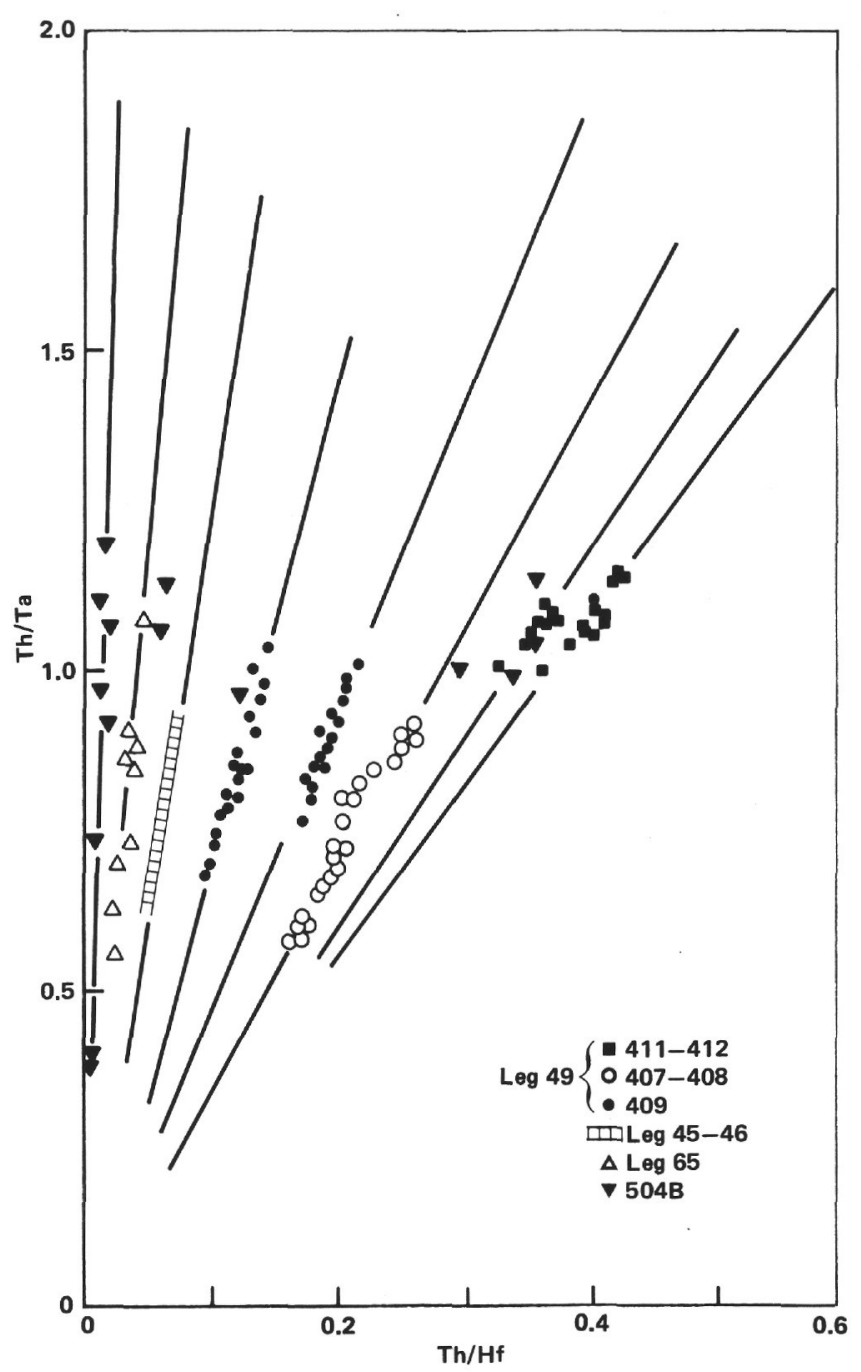

Figure 11. Th/Ta ratio vs. Th/Hf ratio for samples from Hole 504B from the Pacific and North Atlantic oceans (Bougault, Treuil, et al., 1979; Bougault, Cambon, et al., 1979; Wood et al., 1979).

ately depleted LREE with $(\mathrm{La} / \mathrm{Sm})_{N}=0.6-0.7$. We note that these samples are also enriched in $\mathrm{Nb}, \mathrm{Ta}$, and $\mathrm{Th}$.

\section{PETROGENETIC CONSIDERATIONS}

Due to the low mineral-melt $K_{D}$ values (Table 6), the REE in melt produced by equilibrium batch melting will be enriched roughly according to the reciprocal of the degree of melting (i.e., $C_{\mathrm{p}} / C_{\mathrm{o}}=1 / F$ ). Thus, the REE pattern of the melt will mimic that of the source $\left[(\mathrm{La} / \mathrm{Sm})_{\mathrm{N}}\right.$ source $(\mathrm{La} / \mathrm{Sm})_{\mathrm{N}}$ liquid and $(\mathrm{Gd} / \mathrm{Yb})_{\mathrm{N}}{ }^{\text {source }}(\mathrm{Gd} /$ $\mathrm{Yb})_{\mathrm{N}}^{\text {liquid] }}$ and the overall abundance of the REE can be used to estimate the percentage of melting. The HREE of the most primitive basalts analyzed from Hole 504B are about 10x chondrites. This leads to an approximation of about $20 \%$ of melting of mantle peridotite, assuming initial values of $2 x$ chondrites. Again, due to generally low $K_{D}$ values, any reasonable degree of fractional crystallization will not substantially alter the REE patterns except for, perhaps, Eu. 
Table 5. REE concentrations (ppm) in Hole 504B basalts.

\begin{tabular}{|c|c|c|c|c|c|c|c|c|c|c|c|c|c|c|}
\hline $\begin{array}{l}\text { Sample } \\
\text { Interval in cm } \\
\text { Piece }\end{array}$ & $\begin{array}{l}8-2 \\
125-131\end{array}$ & $\begin{array}{l}18-1 \\
84-98\end{array}$ & $\begin{array}{l}19-1 \\
73-77\end{array}$ & $\begin{array}{l}44-1 \\
25-28\end{array}$ & $\begin{array}{l}56-1 \\
139-148\end{array}$ & $\begin{array}{l}57-1 \\
51-55\end{array}$ & $\begin{array}{l}72-3 \\
41-43 \\
5 a\end{array}$ & $\begin{array}{l}87-1 \\
93-95 \\
6 \mathrm{~d}\end{array}$ & $\begin{array}{l}88-1 \\
48-50 \\
2 \mathrm{~d}\end{array}$ & $\begin{array}{l}91-1 \\
134-137 \\
18\end{array}$ & $\begin{array}{l}108-1 \\
57-60 \\
7\end{array}$ & $\begin{array}{l}111-1 \\
86-89 \\
9 \\
\end{array}$ & $\begin{array}{l}136-1 \\
35-60 \\
5\end{array}$ & $\begin{array}{c}137-1 \\
41-47 \\
5\end{array}$ \\
\hline $\mathrm{La}$ & $\begin{array}{l}+ \text { n.d. } \\
1.09\end{array}$ & $\begin{array}{l}6.10 \\
6.71\end{array}$ & $\begin{array}{l}7.90 \\
8.18\end{array}$ & $\begin{array}{l}0.78 \\
1.03\end{array}$ & $\begin{array}{l}3.20 \\
3.47\end{array}$ & $\begin{array}{l}2.90 \\
3.27\end{array}$ & $\begin{array}{l}0.89 \\
0.91\end{array}$ & $\begin{array}{l}1.03 \\
1.19\end{array}$ & $\begin{array}{l}2.75 \\
2.75\end{array}$ & $\begin{array}{l}0.52 \\
0.76\end{array}$ & $\begin{array}{l}0.93 \\
0.92\end{array}$ & $\begin{array}{l}1.36 \\
1.49\end{array}$ & $\begin{array}{l}1.24 \\
1.28\end{array}$ & $\begin{array}{l}0.76 \\
1.00\end{array}$ \\
\hline $\mathrm{Ce}$ & $\begin{array}{l}+ \text { n.d. } \\
4.02\end{array}$ & $\begin{array}{c}\text { n.d } \\
15.09\end{array}$ & $\begin{array}{c}\text { n.d } \\
18.08\end{array}$ & $\begin{array}{l}\text { n.d } \\
4.01\end{array}$ & $\begin{array}{c}\text { n.d } \\
10.66\end{array}$ & $\begin{array}{c}\text { n.d } \\
10.47\end{array}$ & $\begin{array}{l}4.6 \\
3.75\end{array}$ & $\begin{array}{l}4.2 \\
4.59\end{array}$ & $\begin{array}{l}8.3 \\
7.62\end{array}$ & $\begin{array}{l}\text { n.d } \\
2.80\end{array}$ & $\begin{array}{l}4 \\
3.51\end{array}$ & $\begin{array}{l}6.7 \\
5.09\end{array}$ & $\begin{array}{l}5 \\
4.87\end{array}$ & $\begin{array}{l}\text { h.d } \\
3.66\end{array}$ \\
\hline $\mathrm{Nd}$ & 4.63 & 9.61 & 10.97 & 4.71 & 9.39 & 9.31 & 4.50 & 5.38 & 6.42 & 3.53 & 4.25 & 5.14 & 5.72 & 4.16 \\
\hline $\mathrm{Sm}$ & 1.87 & 2.82 & 3.12 & 1.99 & 3.16 & 3.18 & 1.85 & 2. & 2.22 & 1.55 & 1.71 & 1.88 & 2.31 & 1.71 \\
\hline Eu & $\begin{array}{l}+ \text { n.d } \\
0.73\end{array}$ & $\begin{array}{l}1.01 \\
1.02\end{array}$ & $\begin{array}{l}1.12 \\
1.11\end{array}$ & $\begin{array}{l}0.85 \\
0.79\end{array}$ & $\begin{array}{l}1.24 \\
1.16\end{array}$ & $\begin{array}{l}1.22 \\
1.15\end{array}$ & $\begin{array}{l}0.74 \\
0.71\end{array}$ & $\begin{array}{l}0.83 \\
0.85\end{array}$ & $\begin{array}{l}0.87 \\
0.79\end{array}$ & $\begin{array}{l}0.68 \\
0.61\end{array}$ & $\begin{array}{l}0.60 \\
0.68\end{array}$ & $\begin{array}{l}0.88 \\
0.75\end{array}$ & $\begin{array}{l}0.95 \\
0.76\end{array}$ & $\begin{array}{l}0.68 \\
0.68\end{array}$ \\
\hline Gd & 2.96 & 3.56 & 3.85 & 3.09 & 4.34 & 4.28 & 2.90 & 3.45 & 2.98 & 2.50 & 2.63 & 2.60 & 3.40 & 2.56 \\
\hline $\mathrm{Tb}$ & + n.d. & 0.57 & 0.62 & 0.54 & 0.72 & 0.72 & 0.48 & 0.52 & 0.54 & 0.43 & 0.68 & 0.49 & 0.68 & 0.45 \\
\hline Dy & 3.82 & 4.16 & 4.33 & 4.20 & 5.30 & 5.35 & 3.61 & 4.44 & 3.80 & 3.36 & 3.30 & 3.30 & 4.48 & 3.26 \\
\hline $\mathrm{Er}$ & 2.54 & 2.55 & 2.75 & 2.83 & 3.43 & 3.43 & 2.37 & 2.93 & 2.37 & 2.26 & 2.15 & 2.15 & 2.89 & 2.10 \\
\hline $\mathrm{Yb}$ & 2.55 & 2.50 & 2.67 & 2.87 & 3.39 & 3.41 & 2.30 & 2.89 & 2.26 & 2.27 & 2.07 & 2.01 & 2.79 & 2.01 \\
\hline $\mathrm{Lu}$ & 0.40 & 0.39 & 0.41 & 0.45 & 0.53 & 0.53 & 0.36 & 0.43 & 0.34 & 0.36 & 0.31 & 0.31 & 0.43 & 0.30 \\
\hline$(\mathrm{La} / \mathrm{Sm})_{\mathrm{N}}$ & 0.36 & 1.45 & 1.60 & 0.32 & 0.66 & 0.63 & 0.30 & 0.33 & 0.75 & 0.30 & 0.33 & 0.48 & 0.34 & 0.36 \\
\hline$(\mathrm{La} / \mathrm{Yb})_{\mathrm{N}}$ & 0.28 & 1.77 & 2.03 & 0.24 & 0.68 & 0.63 & 0.26 & 0.27 & 0.80 & 0.22 & 0.24 & 0.49 & 0.30 & 0.33 \\
\hline $\begin{array}{l}(\mathrm{Gd} / \mathrm{Yb})_{\mathrm{N}} \\
(+\mathrm{NAA})\end{array}$ & 0.93 & 1.14 & 1.16 & 0.87 & 1.03 & 1.00 & 1.02 & 0.96 & 1.06 & 0.88 & 1.02 & 1.04 & 0.98 & 1.02 \\
\hline
\end{tabular}
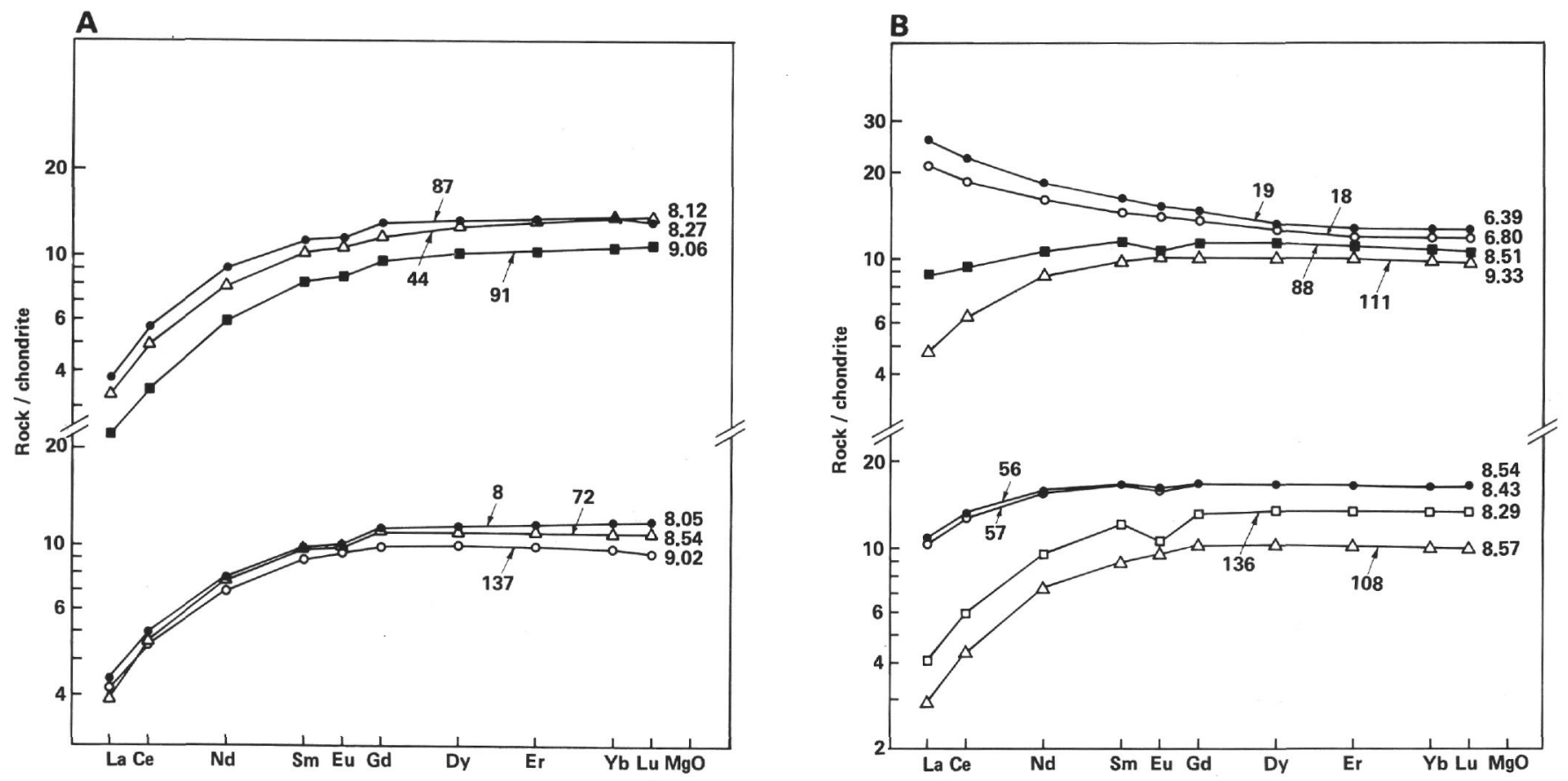

Figure 12. Rare earth patterns for 14 selected samples from Hole 504B (Legs 69, 70, and 83). The chondrite normalization values are from Masuda (1973), divided by 1.2 .

From the REE patterns, together with the $\mathrm{MgO}$ data and refractory trace element distribution seen earlier, we may conclude that the mantle sources are not homogeneous. There are zones enriched in LREE that served as the source for the basalts from Unit 5. However, most source regions are strongly depleted in LREE and other characteristic elements such as $\mathrm{Ta}, \mathrm{Nb}$, and $\mathrm{Th}$.

The effect of fractional crystallization on REE distribution may be examined in Figure 13. These rocks have relatively high $\mathrm{MgO}(6.3-9.5$, average 8.5$)$ and $\mathrm{mg}$ val- 
Table 6. Distribution coefficient values.

\begin{tabular}{llll}
\hline \multicolumn{1}{c}{ Mineral } & La & \multicolumn{1}{c}{ Sm } & \multicolumn{1}{c}{ Yb } \\
\hline Clinopyroxene & 0.07 & 0.26 & 0.30 \\
Olivine & 0.01 & 0.011 & 0.02 \\
Plagioclase & 0.08 & 0.05 & 0.025 \\
Garnet & 0.005 & 0.15 & 4.0 \\
\hline
\end{tabular}

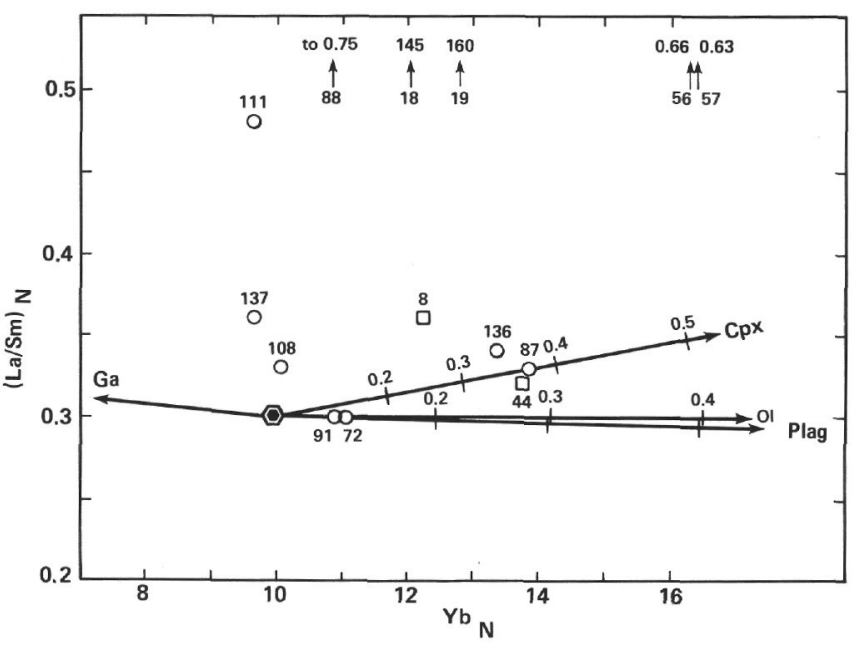

Figure 13. $(\mathrm{La} / \mathrm{Sm})_{\mathrm{N}}$ vs. $(\mathrm{Yb})_{\mathrm{N}}$ variation diagram showing monomineralic fractionation from a hypothetical parental liquid.

ues $(0.61-0.70)$. This suggests that the liquids may not have undergone a very significant fractional crystallization. Some samples have negative Eu anomalies, probably the result of plagioclase fractionation. Using $\mathrm{K}_{\mathrm{D} \mathrm{Eu}}{ }^{\text {plg } / \mathrm{liq}}=0.55, \mathrm{fO}_{2}=10^{8}$ (Drake and Weill, 1975), Eu/Eu* $=0.81$ (Sample 136) can be accounted for by $30 \%$ of plagioclase fractionation from a primitive basalt with $\mathrm{Eu} / \mathrm{Eu}^{*}=1$ (Sample 108). $\mathrm{Ni}$ and $\mathrm{Cr}$ concentrations suggest that olivine and clinopyroxene are also phases of fractionation. We have chosen a primary liquid $(\mathrm{P})$ with $\mathrm{Yb}=10 \mathrm{x}$ chondrites and $(\mathrm{La} / \mathrm{Sm})_{\mathrm{N}}=0.3$. Thus, olivine and/or plagioclase fractionation can only enhance the REE concentrations in the liquid's residuals, but not change their $\mathrm{La} / \mathrm{Sm}$ ratios appreciably. Clinopyroxene is capable of changing the $\mathrm{La} / \mathrm{Sm}$ ratios, but given a reasonable amount of crystal fractionation, the wide spread in $\mathrm{La} / \mathrm{Sm}$ ratios observed cannot be adequately explained. Therefore, crystal fractionation involving the common phases will not effectively fractionate the REE patterns. Consequently, the exceptional chemical characteristics of Units 5, 36, and 69 must suggest their derivation from a distinct source. Even if only samples with highly depleted LREE patterns are considered, the variation of $\mathrm{La} / \mathrm{Sm}$ ratios cannot be accounted for by the fractional crystallization process of a single parental magma. Although there is close similarity in major element chemistry, there is not such coherence in trace element abundances. Such differences are presumed to reflect heterogeneity in the mantle source or in magma generating processes.
Magma mixing (Rhodes, Dungan, et al., 1979) may explain several enigmatic features of ocean floor basalt petrogenesis not readily understandable in terms of the crystal fractionation process alone. The hypothesis of "dynamic melting" (Langmuir et al., 1977) of a homogeneous source region can explain the different REE patterns within a single hole. This process can produce different melts from a homogeneous source that has a different degree of LREE enrichment and crossing REE patterns. This is observed for FAMOUS area samples (DSDP Leg 37).

Among the basaltic rocks drilled in Hole 504B, only three (Units 5, 36, and 69) of the 167 units have exceptional trace element chemistry. It is likely that more than three units with exceptional chemistry would have been detected had a higher percentage of the drilled core been recovered (Legs 69, 70, and 83 penetrated $1075 \mathrm{~m}$ of oceanic basement with a total recovery of $224 \mathrm{~m}$, an average of only $20.8 \%$ ).

\section{CONCLUSIONS}

1. The basement rocks recovered by the drilling of Hole 504B consist of basaltic rocks formed by a number of discrete eruptive and intrusive events. In their major bulk composition, these rocks are rather uniform from the top to the bottom of the hole. No chemical distinctions can be made between lava flows that make up the upper (Legs 69 and 70) part of the basement and dikes that prevail in the lower (Leg 83) part.

2. Most of the Hole 504B samples have relatively high mg values suggesting that they are rather primitive and had experienced only a small degree of fractional crystallization before their eruption or intrusion. The observation of major elements and compatible trace element data suggests that the relative importance of mineral fractionation is plagioclase, followed by olivine, clinopyroxene, and spinel.

3. These basalts are very depleted in the most incompatible or hygromagmaphile elements ( $\mathrm{Th}, \mathrm{Ta}$, and $\mathrm{Nb}$ ) and show very LREE-depleted patterns.

4. Within the $1075.5 \mathrm{~m}$ of basement cored, only three units (namely Units 5, 36, and 69 with a combined length of $35 \mathrm{~m}$ ) differ significantly in trace element chemistry from all other rocks. They show LREE-enriched or slightly depleted patterns in the same way as most hygromagmaphile elements ( $\mathrm{Th}, \mathrm{Ta}$, and $\mathrm{Nb}$ ). For Units 5 and 36, $\mathrm{TiO}_{2}$ and $\mathrm{P}_{2} \mathrm{O}_{5}$ concentrations are also higher.

5. Magmatic mixing, as advocated by Rhodes, Dungan, et al. (1979), can account for some of the variations observed. Neither partial melting nor fractional crystallization processes could account for all the REE and other hygromagmaphile element characteristics of these rocks. This implies that the liquids are probably derived from heterogeneous sources.

\section{REFERENCES}

Akella, J., Williams, R. J., and Mullins, O. 1976. Solubility of Cr, Ti, and $\mathrm{Al}$ in co-existing olivine, spinel, and liquid at $1 \mathrm{~atm}$. Proc. Seventh Lunar Sci. Conf., 1179-1194.

Anderson, R. N., Honnorez, J., Becker, K., Adamson, A. C., Alt, J. C., Emmermann, R., Kempton, P. D., Kinoshita, H., Laverne, C., 
Mottl, M. J., and Newmark, R. L., 1982. DSDP Hole 504B, the first reference section over $1 \mathrm{~km}$ through Layer $2 \mathrm{c}$ of the oceanic crust. Nature, 300:589-594.

Autio, L. K., and Rhodes, J. M., 1983. Costa Rica Rift zone basalts: geochemical and experimental data from a possible example of multistage melting. In Cann, J. R., Langseth, M. G., Honnorez, J., Von Herzen, R. P., White S. M., et al., Init. Repts. DSDP, 69: Washington, (U.S. Govt. Printing Office), 729-746.

Bender, J. F., Hodges, F. N., and Bence, A. E., 1978. Petrogenesis of basalts from the project FAMOUS area: Experimental study from 0-15 kbars. Earth Planet. Sci. Lett., 41:277-302.

Bird, M. L., 1971. Distribution of trace elements in olivines and pyroxenes-an experimental study [Ph.D. dissert.]. Univ. of Missouri-Rolla.

Blanchard, D. P., Rhodes, J. M., Dungan, M. A., Rodgers, K. V., Donaldson, C. H., Brannon, J. C., Jacobs, J. W., and Gibson, E. K., 1976. The chemistry of petrology of basalts from Leg 37 of the Deep Sea Drilling Project. J. Geophys. Res., 81:4231-4246.

Bougault, H., 1977. Major elements: Analytical chemistry on broad and preliminary results. In Aumento, F., Melson, W. G., et al., Init. Repts. DSDP, 37: Washington (U.S. Govt. Printing Office), 643-651.

1980. Contribution des éléments de transition à la compréhension de la génèse des basaltes océaniques; analyse des éléments tracés dans les roches par spectrometrie de fluorescence $\mathrm{X}$ [Thèse d'état]. Université de Paris VII.

Bougault, H., Cambon, P., Corre, O., Joron, J. L., and Treuil, M., 1979. Evidence for variability of magmatic processes and upper mantle heterogeneity in the axial region of the Mid-Atlantic Ridge near $22^{\circ} \mathrm{N}$ and $36^{\circ} \mathrm{N}$. Tectonophysics, 55:11-34.

Bougault, H., Cambon, P., and Toulhoat, H., 1977. X-ray spectrometric analysis of trace elements in rocks. Correction for Instrumental Interferences. X-Ray Spectrometry, 6:66-72.

Bougault, H., and Hekinian, R., 1974. Rift valley in the Atlantic Ocean near $36^{\circ} 50^{\prime} \mathrm{N}$ : Petrology and geochemistry of basaltic rocks. Earth Planet. Sci. Lett., 24:249-261.

Bougault, H., Joron, J. L., and Treuil, M., 1979. Alteration fractional crystallization, partial melting, mantle properties from trace elements in basalts recovered in the North Atlantic. In Talwani, M., Harrison, C. G., and Hayes, D. E. (Eds.), Deep Drilling Results in the Atlantic Ocean: Ocean Crust: Washington (Am. Geophys. Un.).

Bougault, H., Treuil, M., and Joron, J. L., 1979. Trace elements in basalts from $23^{\circ} \mathrm{N}$ and $36^{\circ} \mathrm{N}$ in the Atlantic Ocean: Fractional crystallization, partial melting, and heterogeneity of the upper mantle. In Melson, W.G., Rabinowitz, P. D., et al., Init. Repts. DSDP, 45: Washington (U.S. Govt. Printing Office), 493-506.

Bryan, W. B., Thompson, G., Frey, F. A., and Dickey, J. S., 1976. Inferred setting and differentiation in basalts from the Deep Sea Drilling Project. J. Geophys. Res., 81:4285-4304.

Cambon, P., Bougault, H., Joron, J. L., and Treuil, M., 1983. Basalts from the East Pacific Rise: An example of typical oceanic crust depleted in hygromagmaphile elements. In Lewis, B. T. R., Robinson, P., et al., Init. Repts. DSDP, 65: Washington (U.S. Govt. Printing Office), 623-633.

Dale, I. M., and Henderson, P., 1972. The partition of transition elements in phenocryst-bearing basalts and the implications about melt structure. Proc. 24th Int. Geol. Conf., Section 10:105-111.

Donaldson, C. H., and Brown, R. W., 1977. Refractory megacrysts and magnesium-rich melt inclusions within spinel in oceanic tholeiites: Indicators of magma mixing and parental magma composition. Earth Planet. Sci. Lett., 37:81-89.

Drake, M. J., and Weill, D. F., 1975. The partition of Sr, Ba, Ca, Y, $\mathrm{Eu}^{2+}, \mathrm{Eu}^{3+}$, and other REE between plagioclase feldspar and magmatic liquid: An experimental study. Geochim. Cosmochim. Acta, 39:689-712.

Duke, J. M., 1976. Distribution of the period four transition elements among olivine, calcic clinopyroxene and mafic silicate liquids: Experimental results. J. Petrol., 17:499-521.

Dungan, M A., and Rhodes, J. M., 1978. Residual glasses and melt inclusions in basalts from DSDP Legs 45 and 46-evidence for magma mixing. Contrib. Mineral. Petrol., 67:417-431.

Etoubleau, J., Corre, O., Joron, J. L., Bougault, H., and Treuil, M., 1983. Costa Rica Rift: Variably depleted basalts in the same hole. In Cann, J. R., Langseth, M. G., Honnorez, J., Von Herzen, R.
P., White, S. M., et al., Init. Repts. DSDP, 69: Washington (U.S. Govt. Printing Office), 765-774.

Etoubleau, J., Bougault, H., Rideout, M., Brannon, J., and Weaver, B., in press. Analysis of trace elements in basalts by X-ray fluorescence spectrometry on board the Glomar Challenger: Leg 82-a discussion for Niobium. In Bougault, H., Cande, S. C., et al., Init. Repts. DSDP, 82: Washington (U.S. Govt. Printing Office).

Evans, B. W., 1969. The nickel partition geothermometer applied to the prehistoric Makaopuhi Lava Lake, Hawaii. Geochim. Cosmochim. Acta, 33:409-411.

Fish, M. R., 1978. Melting relations and mineral chemistry of Iceland and Reykjanes Ridge basalts. [Ph.D. dissert.] Grad. School of Oceanography, Univ. of Rhode Island, Kingston, R. I.

Frey, F. A., Bryan, W. B., and Thompson, G., 1974. Atlantic Ocean floor: Geochemistry and petrology of basalts from Legs 2 and 3 of the Deep Sea Drilling Project. J. Geophys. Res., 79:5507-5527.

Frey, F. A., Green, D. H., and Roy, S. D., 1978. Integrated models of basalt petrogenesis: A study of quartz tholeiites to olivine melilitites from southeastern Australia utilizing geochemical and experimental petrological data. J. Petrol., 19:463-513.

Gast, P. W., 1968. Trace element fractionation and the origin of tholeiitic and alkaline magma types. Geochim. Cosmochim. Acta, 32: 1057-1086.

Haku, T. A., and Wright, T. L., 1967. The fractionation of nickel between olivine and augite as a geothermometer. Geochim. Cosmochim. Acta, 31:877-884.

Hart, S. R., and Davis, K. E., 1978. Nickel partitioning between olivine and silicate melt. Earth Planet. Sci. Lett., 40:203-219.

Hubberton, H.-W., Emmermann, R., and Puchelt, H., 1983. Geochemistry of basalts from Costa Rica Rift Sites 504 and 505 (Deep Sea Drilling Project Legs 69 and 70). In Cann, J. R., Langseth, M. G., Honnorez, J., Von Herzen, R. P., White, S. M., et al., Init. Repts. DSDP, 69: Washington (U.S. Govt. Printing Office), 791-804.

Jaffrezic, H., Joron, J. L., and Treuil, M., 1977. Trace element determination in rock powder. A study of the precision for a given analytical procedure. Instrumental epithermal neutron activation. $J$. Radioanal. Chem., 39:185-188.

Jahn, B. M., Auvray, B., Blais, S., Capdevila, R., Cornichet, J., Vidal, F., and Hameurt, J., 1980. Trace element geochemistry and petrogenesis of Finnish greenstone belts. J. Petrol., 21:201-244.

Jahn, B. M., Griffiths, B. J., Charlot, R., Cornichet, J., and Vidal, F., 1980. $\mathrm{Nd}$ and $\mathrm{Sr}$ isotopic compositions and REE abundances of Cretaceous MORB (Holes 417 and 418A, Legs 51, 52, and 53). Earth Planet. Sci. Lett., 48:171-184.

Johnson, J. R., 1979. Transitional basalts and tholeiites from the East Pacific Rise $9^{\circ}$ N. J. Geophys. Res., 84:1635-1652.

Joron, J. L., Briqueu, L., Bougault, H., and Treuil, M., 1980. East Pacific Rise, Galapagos Spreading Center and Siqueiros Fracture Zone, Deep Sea Drilling Project Leg 54: Hygromagmaphile elements-A comparison with the North Atlantic. In Rosendahl, B., Hekinian, R., et al., Init. Repts. DSDP, 54: Washington (U.S. Govt. Printing Office), 725-735.

Joron, J. L., Treuil, M., Jaffrezic, H., Villemant, B., and Richard, O., 1980. Geochimie des éléments en traces du magmatisme de l'Afar et de la megastructure. Mer Rouge-Afar-Golfe d'Aden. Implications pétrogénétiques et géodynamiques. Bull. Soc. Geol. France, 7, 22(6):945-957.

Kudo, A. M., Barker, S. E., Keil, K., and Besson, M. H., 1982. Geochemical modeling of basalts from DSDP Leg 65, East Pacific Rise, Gulf of California. Geochim. Cosmochim. Acta, 46: 2427-2434.

Langmuir, C. H., Bender, J. F., Bense, A. E., and Hanson, G. N., 1977. Petrogenesis of basalts from the FAMOUS area: Mid-Atlantic Ridge. Earth Planet. Sci. Lett., 36:133-156.

Leeman, W. P., 1974. Part I. Petrology of basaltic lavas from the Snake River Plain, Idaho; Part II. Experimental determination of partitioning of divalent cations between olivine and basaltic liquid [Ph.D. dissert.]. Univ. of Oregon, Eugene, OR.

Lindstrom, D. J., and Weill, D. F., 1978. Partitioning of transition metals between diopside and coexisting silicate liquids. I. Nickel, cobalt, and manganese. Geochim. Cosmochim. Acta, 42:817-831.

Marsh, N. G., Tarney, J., and Hendry, G. L., 1983. Trace element geochemistry of basalts from Hole 504B, Panama Basin, Deep Sea Drilling Project Legs 69 and 70. In Cann, J. R., Langseth, M. G., 
Honnorez, J., Von Herzen, R. P., White, S. M., et al., Init. Repts. $D S D P$, 69: Washington (U.S. Govt. Printing Office), 747-764.

Masuda, A., Kanamura, N., and Tanaka, T., 1973. Fine structure of mutually normalized rare earth patterns of chondrites. Geochim. Cosmochim. Acta, 37:239-248.

Muir, I. D., and Tilley, C. E., 1964. Basalts from the northern part of the rift zone of the Mid-Atlantic Ridge. J. Petrol., 5:409-434.

Mysen, B. O., and Kushiro, I., 1976. Partitioning of iron, nickel, and magnesium between metal, oxides, and silicates in Allende meteorite as a function of $\mathrm{FO}_{2}$. Yearbook, Carnegie Inst. Wash., 75: 678-684.

Natland, J. H., Adamson, A. C., Laverne, C., Melson, W. G., and O'Hearn, T., 1983. A compositionally nearly steady-state magma chamber at the Costa Rica Rift: Evidence from basalt glass and mineral data, Deep Sea Drilling Project Sites 501, 504 and 505. In Cann, J. R., Langseth, M. G., Honnorez, J., Von Herzen, R. P., White, S. M., et al., Init. Repts. DSDP, 69: Washington (U.S. Govt. Printing Office), 811-858.

Onuma, N., Higuchi, H., Wakita, H., and Nagasawa, H., 1968. Trace element partition between two pyroxenes and the host lava. Earth Planet. Sci. Lett., 5:47-51.

Rhodes, J. M., Blanchard, D. P., Dungan, M. A., Rodgers, K. V., and Brannon, J. C., 1979. Chemistry of Leg 45 basalts. In Melson, W. G., Rabinowitz, P. D., et al., Init. Repts. DSDP, 45: Washington (U.S. Govt. Printing Office), 447-460.

Rhodes, J. M., Dungan, M. A., Blanchard, D. P., and Long, P. E., 1979. Magma mixing at mid-ocean ridges: Evidence from basalts drilled near $22^{\circ} \mathrm{N}$ on the Mid-Atlantic Ridge. Tectonophysics, 55: 35-62.

Schilling, J. G., 1975. Rare earth variations across "normal sections" of the Reykjanes Ridge, $60^{\circ}-53^{\circ} \mathrm{N}$, Mid-Atlantic Ridge, $2^{\circ}-19^{\circ} \mathrm{S}$, and evidence on the composition of the underlying low-velocity layer. J. Geophys. Res., 80:1459-1473.

Schreiber, H. D., and Haskin, L. A., 1976. Chromium in basalts: Experimental determination of redox states and partitioning among synthetic silicate phases. Proc. Seventh Lunar Sci. Conf., 1221-1259.
Shaw, D. M., 1970. Trace element fractionation during anatexis. Geochim. Cosmochim. Acta, 34:237-243.

Sun, S. S., and Nesbitt, R. W., 1978. Petrogenesis of Archaean ultrabasic and basic volcanics: Evidence from rare earth elements. Contrib. Mineral. Petrol., 65:301-325.

Sun, S. S., Nesbitt, R. W., and Sharaskin, A. Y., 1979. Geochemical characteristics of mid-ocean ridge basalts. Earth Planet. Sci. Lett., 44:119-138.

Taylor, S. R., Kaye, M., White, A. J. R., Duncan, A. R., and Ewart, A., 1969. Genetic significance of $\mathrm{Co}, \mathrm{Cr}, \mathrm{Ni}, \mathrm{Sc}$, and $\mathrm{V}$ content of andesites. Geochim. Cosmochim. Acta, 33:275-286.

Thompson, G., Shido, F., and Miyashiro, A., 1972. Trace element distributions in fractionated oceanic basalts. Chem. Geol., 9:89-97.

Wager, L. R., and Mitchell, R. L., 1951. The distribution of trace elements during strong fractionation of basic magma-a further study of the Skaengaard intrusion, East Greenland. Geochim. Cosmochim. Acta, 1:129-208.

Walker, D., Shibata, T., and de Long, S. E., 1979. Abyssal tholeiites from the Oceanographer Fracture Zone. II. Phase equilibria and mixing. Contrib. Mineral. Petrol., 70:111-125.

White, W. M., and Schilling, J. G., 1978. The nature of the origin of geochemical variation in Mid-Atlantic Ridge basalts from the central North Atlantic. Geochim. Cosmochim. Acta, 42:1501-1516.

Wilkinson, J. F. G., 1982. The genesis of mid-ocean ridge basalt. Earth Sci. Rev., 18:1-57.

Wood, D. A., Tarney, J., Varet, J., Saunders, A. D., Bougault, H., Joron, J. L., Treuil, M., and Cann, J. R., 1979. Geochemistry of basalts drilled in the North Atlantic by IPOD Leg 49: Implications for mantle heterogeneity. Earth Planet. Sci. Lett., 43:77-97.

Yoder, H. S., and Tilley, C. E., 1962. Origin of basalt magmas: An experimental study of natural and synthetic rock systems. J. Petrol., 3:342-532.

Date of Initial Receipt: 27 September 1983 Date of Acceptance: 15 March 1984 